\title{
New Types of $F$-Contraction for Multivalued Mappings and Related Fixed Point Results in Abstract Spaces
}

\author{
Shaif Saleh Alshoraify, ${ }^{1}$ Abdullah Shoaib $\mathbb{D D}^{2},{ }^{2}$ and Muhammad Arshad ${ }^{1}{ }^{1}$ \\ ${ }^{1}$ Department of Mathematics and Statistics, International Islamic University, H-10, Islamabad 44000, Pakistan \\ ${ }^{2}$ Department of Mathematics and Statistics, Riphah International University, Islamabad, Pakistan \\ Correspondence should be addressed to Abdullah Shoaib; abdullahshoaib15@yahoo.com
}

Received 26 April 2019; Revised 7 June 2019; Accepted 23 June 2019; Published 16 July 2019

Academic Editor: Gestur Ólafsson

Copyright (c) 2019 Shaif Saleh Alshoraify et al. This is an open access article distributed under the Creative Commons Attribution License, which permits unrestricted use, distribution, and reproduction in any medium, provided the original work is properly cited.

In this article, we establish fixed point results for a pair of multivalued mappings satisfying generalized contraction on a sequence in dislocated $b$-quasi metric spaces and $F \rho_{s}^{*}$ Khan type contraction on a sequence in $b$-quasi metric spaces. An example and an application have been discussed. Our results modify and generalize many existing results in literature.

\section{Introduction and Preliminaries}

A point $v$ is said to be a fixed point of a multivalued/selfmapping $E$, if $v \in E v / v=E v$. Fixed point theory has a large number of applications, for example, [1-4]. Czewick [5] initiated the study of fixed point in b-metric spaces. Many authors used the concept of b-metric spaces to prove the existence and the uniqueness of a fixed point for several contraction mappings [6-9]. Furthermore, dislocated quasimetric spaces [10-13] generalized abstract spaces such as dislocated metric spaces [14] and quasi-metric spaces [15-17]. Recently, Klin-eam and Suanoom [18] introduced the concept of dislocated $b$-quasi metric spaces. Fixed point results in complete dislocated $b$-quasi metric spaces can be seen in $[19,20]$.

Wardowski [21] generalized many fixed point results in a beautiful way by introducing $F$-contraction (see also [6, 22-30]). Nadler [31] extended Banach's contraction mapping principle to a fundamental fixed point theorem for multivalued mappings. Since then, an interesting and rich fixed point theory for such mappings was developed in many directions; see [32-36]. The results of single valued mappings can be generalized by using multivalued mappings. Results for multivalued mappings have applications in engineering, Nash equilibria, and game theory [37-40]. Rasham et al. [41] obtained fixed point results for a pair of multivalued
F-contractive mappings, which are extensions of some multivalued fixed point results.

This paper introduces new types of $F$-contractions on a sequence and generalizes many recent results. An example has been given to show how our results are valid when the others fail. An application has been given to obtain a solution of a system of integral equations.

Definition 1 (see [18]). Let $Y$ be a nonempty set and $s \geq 1$ a real number. A mapping $d_{q b}: Y \times Y \rightarrow[0, \infty)$ is called a dislocated quasi $b$-metric (or simply $d_{q b}$-metric), if the following conditions hold for any $x, y, z \in Y$ :

(a) If $d_{q b}(x, y)=d_{q b}(y, x)=0$, then $x=y$;

(b) $d_{q b}(x, y) \leq s\left[d_{q b}(x, z)+d_{q b}(z, y)\right]$.

The pair $\left(Y, d_{q b}\right)$ is called a dislocated quasi $b$-metric space (in short dislocated $b$-quasi-metric space).

The following remarks can be observed:

(a) If $s=1$, then a dislocated $b$-quasi-metric space becomes a dislocated quasi-metric space [12];

(b) if $s=1$ and $x=y$ implies $d_{q b}(x, y)=d_{q b}(y, x)=0$, then $\left(Y, d_{q b}\right)$ becomes a quasi-metric space [17];

(c) if $d_{q b}(x, y)=d_{q b}(y, x)$ and $x=y$ implies $d_{q b}(x, y)=$ 0 , then $\left(Y, d_{q b}\right)$ becomes a $b$-metric space [9]. 
Example 2 (see [20], let $Y=R^{+}$and $p>1$ ). Define $d_{q b}$ : $Y \times Y \longrightarrow R^{+}$by $d_{q b}(x, y)=|x-y|+|x|$ for $x, y \in X$. Then $\left(Y, d_{q b}\right)$ is a $d_{q b}$-metric space with $s=2^{p}>1$. But it is not a quasi $b$-metric space. Also it is not a dislocated $b$-metric space. It is obvious that $\left(Y, d_{q b}\right)$ is neither $b$-metric space nor dislocated quasi-metric space.

Definition 3 (see [11]). Let $\left(Y, d_{q b}\right)$ be a dislocated $b$-quasimetric space. Let $\left\{y_{n}\right\}$ be a sequence in $\left(Y, d_{q b}\right)$, and then

(a) $\left\{y_{n}\right\}$ is called Cauchy if $\forall \varepsilon>0, \exists n_{0} \in N$ such that $\forall n>m \geq n_{0}$ (respectively $\left.\forall m>n \geq n_{0}\right), d_{q b}\left(y_{m}, y_{n}\right)<\varepsilon$.

(b) $\left\{y_{n}\right\}$ dislocated quasi b-converges (for short $d_{q b}$-converges) to $y \in Y$, if $\lim _{n \longrightarrow \infty} d_{q b}\left(y_{n}, y\right)=$ $\lim _{n \rightarrow \infty} d_{q b}\left(y, y_{n}\right)=0$ or for any $\varepsilon>0$, there exists $n_{0} \in N$, such that for all $n>n_{0}, d_{q b}\left(y, y_{n}\right)<\varepsilon$ and $d_{q b}\left(y_{n}, y\right)<\varepsilon$. In this case $y$ is called a $d_{q b}$-limit of $\left\{y_{n}\right\}$.

(c) $\left(Y, d_{q b}\right)$ is called complete if every Cauchy sequence in $Y$ converges to a point $y \in Y$.

Definition 4 (see [12]). Let $\left(Y, d_{q b}\right)$ be a dislocated $b$-quasi metric space. Let $K$ be a nonempty subset of $Y$ and let $x \in Y$. An element $y_{0} \in K$ is called a best approximation in $K$ if

$$
\begin{aligned}
& d_{q b}(x, K)=d_{q b}\left(x, y_{0}\right) \\
& \text { where } d_{q b}(x, K)=\inf _{y \in K} d_{q b}(x, y)
\end{aligned}
$$

and $d_{q b}(K, x)=d_{q b}\left(y_{0}, x\right)$

$$
\text { where } d_{q b}(K, x)=\inf _{y \in K} d_{q b}(y, x) .
$$

If each $x \in Y$ has at least one best approximation in $K$, then $K$ is called a proximinal set.

It is clear that if $d_{q b}(x, K)=d_{q b}(K, x)=0$, then $x \in K$. But if $x \in K$, then $d_{q b}(x, K)$ or $d_{q b}(K, x)$ may not equal zero. We denote $P(Y)$ by the set of all proximinal subsets of $Y$.

Definition 5 (see [12]). The function $H_{d_{q}}: P(Y) \times P(Y) \longrightarrow$ $\mathbb{R}_{+}$, defined by

$$
H_{d_{q b}}(A, B)=\max \left\{\sup _{a \in A} d_{q}(a, B), \sup _{b \in B} d_{q b}(A, b)\right\}
$$

is called dislocated quasi Hausdorff $b$ metric on $P(Y)$. Also $\left(P(Y), H_{d_{q b}}\right)$ is known as dislocated quasi Hausdorff $b$-metric space, where $P(Y)$ is the proximinal subset of $Y$.

Ali et al. [6] extended the family of mapping $\mathscr{F}$ defined by [21] to the family $\mathscr{F}_{S}$ of all functions $F: \mathbb{R}_{+} \longrightarrow \mathbb{R}$ such that

(F1) $F$ is strictly increasing, that is, for all $x, y \in \mathbb{R}_{+}$such that $x<y$ implies $F(x)<F(y)$;

(F2) for each sequence $\left\{\vartheta_{n}\right\}_{n=1}^{\infty}$ of positive numbers, $\lim _{n \rightarrow \infty} \vartheta_{n}=0$ if and only if $\lim _{n \rightarrow \infty} F\left(\vartheta_{n}\right)=-\infty$;

(F3) there exists $k \in(0,1)$ such that $\lim _{\vartheta \longrightarrow 0^{+}} \vartheta^{k} F(\vartheta)=0$.

(F4) For each sequence $\left\{\vartheta_{n}\right\}$ of positive real numbers and such that $\tau+F\left(s \vartheta_{n}\right) \leq F\left(\vartheta_{n-1}\right)$ for each $n \in \mathbb{N}$, and some $\tau>0$, we have $\tau+F\left(s^{n} \vartheta_{n}\right) \leq F\left(s^{n-1} \vartheta_{n-1}\right)$, for each $n \in \mathbb{N}$.
Lemma 6. Let $\left(Y, d_{q b}, s\right)$ be a dislocated b-quasi-metric space. Let $\left(P(Y), H_{d_{q b}}\right)$ be the dislocated quasi Hausdorff b-metric space on $P(Y)$. Then, for all $A, B \in P(Y)$ and for each $a \in A$, there exists $b_{a} \in B$, such that $H_{d_{q b}}(A, B) \geq d_{q b}\left(a, b_{a}\right)$ and $H_{d_{q}}(B, A) \geq d_{q b}\left(b_{a}, a\right)$, where $d_{q b}(a, B)=d_{q b}\left(a, b_{a}\right)$ and $d_{q b}(B, a)=d_{q b}\left(b_{a}, a\right)$.

Lemma 7 (see [6]). Let $\left(Y, d_{b}, s\right)$ be a b-metric space and let $\left\{y_{n}\right\}$ be any sequence in $Y$ for which there exist $\tau>0$ and $F \in$ $\mathscr{F}_{S}$ such that $\tau+F\left(s d_{q b}\left(y_{n}, y_{n+1}\right)\right) \leq F\left(d_{q b}\left(y_{n-1}, y_{n}\right)\right), n \in \mathbb{N}$. Then $\left\{y_{n}\right\}$ is a Cauchy sequence in $Y$.

Lemma 8. Let $\left(X, d_{q b}, s\right)$ be a dislocated b-quasi metric space, and let $\left\{x_{n}\right\}$ be any sequence in $X$ for which there exist $\tau>0$ and $F \in \mathscr{F}_{S}$ such that

$$
\begin{array}{r}
\tau+F\left(s \max \left\{d_{q b}\left(y_{n}, y_{n+1}\right), d_{q b}\left(y_{n+1}, y_{n}\right)\right\}\right) \\
\leq F\left(\max \left\{d_{q b}\left(y_{n-1}, y_{n}\right), d_{q b}\left(y_{n}, y_{n-1}\right)\right\}\right)
\end{array}
$$

for each $n \in \mathbb{N}$. Then $\left\{y_{n}\right\}$ is a Cauchy sequence in $X$.

Proof. Let $\vartheta_{n}=\max \left\{d_{q b}\left(y_{n}, y_{n+1}\right), d_{q b}\left(y_{n+1}, y_{n}\right)\right\}$, for each $n \in$ $\mathbb{N}$. Thus, by (3) and property (F4), we get

$$
\tau+F\left(s^{n} \vartheta_{n}\right) \leq F\left(s^{n-1} \vartheta_{n-1}\right), \quad n \in \mathbb{N} .
$$

Following similar arguments as given in [6], we obtain $\left\{y_{n}\right\}$ is a Cauchy sequence in $X$.

\section{Main Result}

Let $\left(Y, d_{q b}\right)$ be a dislocated $b$-quasi metric space, $y_{0} \in Y$ and $S, T: Y \longrightarrow P(Y)$ be multifunctions on $Y$. Let $y_{1} \in S y_{0}$ be an element such that $d_{q b}\left(y_{0}, S y_{0}\right)=d_{q b}\left(y_{0}, y_{1}\right), d_{q b}\left(S y_{0}, y_{0}\right)=$ $d_{q b}\left(y_{1}, y_{0}\right)$. Let $y_{2} \in T y_{1}$ be such that $d_{q b}\left(y_{1}, T y_{1}\right)=$ $d_{q b}\left(y_{1}, y_{2}\right), d_{q b}\left(T y_{1}, y_{1}\right)=d_{q b}\left(y_{2}, y_{1}\right)$. Let $y_{3} \in S y_{2}$ be such that $d_{q b}\left(y_{2}, S y_{2}\right)=d_{q b}\left(y_{2}, y_{3}\right)$ and so on. Thus, we construct a sequence $y_{n}$ of points in $Y$ such that $y_{2 n+1} \in S y_{2 n}$ and $y_{2 n+2} \in$ $T y_{2 n+1}$, with $d_{q b}\left(y_{2 n}, S y_{2 n}\right)=d_{q b}\left(y_{2 n}, y_{2 n+1}\right), d_{q b}\left(S y_{2 n}, y_{2 n}\right)=$ $d_{q b}\left(y_{2 n+1}, y_{2 n}\right)$, and $d_{q b}\left(y_{2 n+1}, T y_{2 n+1}\right)=d_{q b}\left(y_{2 n+1}, y_{2 n+2}\right)$, $d_{q b}\left(T y_{2 n+1}, y_{2 n+1}\right)=d_{q b}\left(y_{2 n+2}, y_{2 n+1}\right)$, where $n=0,1,2, \cdots$. We denote this iterative sequence by $\left\{T S\left(y_{n}\right)\right\}$. We say that $\left\{T S\left(y_{n}\right)\right\}$ is a sequence in $Y$ generated by $y_{0}$. If $T=S$, then we say that $\left\{X T\left(y_{n}\right)\right\}$ is a sequence in $Y$ generated by $y_{0}$.

Let us introduce the following definition:

Definition 9. Let $\left(Y, d_{q b}, s\right)$ be a dislocated $b$-quasi-metric space and $S, T: Y \longrightarrow P(Y)$ be two multivalued mappings. The pair $(S, T)$ is called a $D Q F$-contraction, if there exists $F \in$ $\mathscr{F}_{S}$ and $\tau, a>0$ such that for every two consecutive points $x, y$ belonging to the range of an iterative sequence $\left\{T S\left(y_{n}\right)\right\}$ with $\max \left\{H_{d_{q b}}(S x, T y), H_{d_{q b}}(T y, S x), D_{q b}(x, y), D_{q b}(y, x)\right\}>$ 0 , we have

$$
\begin{aligned}
\tau+ & \max \left\{F\left(s H_{d_{q b}}(S x, T y)\right), F\left(s H_{d_{q b}}(T y, S x)\right)\right\} \\
& \leq \min \left\{F\left(D_{q b}(x, y)\right), F\left(D_{q b}(y, x)\right)\right\}
\end{aligned}
$$


where

$$
\begin{aligned}
& D_{q b}(x, y)=\max \left\{d_{q b}(x, y),\right. \\
& \frac{d_{q b}(x, S x) \cdot d_{q b}(y, T y)}{a+\max \left\{d_{q b}(x, y), d_{q b}(y, x)\right\}}, d_{q b}(x, S x), \\
& \left.d_{q b}(y, T y)\right\},
\end{aligned}
$$

And we now prove the following main result.

Theorem 10. Let $\left(Y, d_{q b}, s\right)$ be a complete dislocated b-quasimetric with $s \geq 1$ and $(S, T)$ be a DQF-contraction. Then $\left\{T S\left(y_{n}\right)\right\} \longrightarrow u \in Y$. Also, if (5) holds for each $x, y \in\{u\}$, then $S$ and $T$ have a common fixed point $u$ in $Y$ and $d_{q b}(u, u)=0$.

Proof. Let $\left\{T S\left(y_{p}\right)\right\}$ be the iterative sequence in $Y$ generated by a point $y_{0} \in Y$. If

$$
\begin{gathered}
\max \left\{H_{d_{q b}}\left(S y_{2 p^{\prime}}, T y_{2 p^{\prime}+1}\right), H_{d_{q b}}\left(T y_{2 p^{\prime}+1}, S y_{2 p^{\prime}}\right),\right. \\
\left.D_{q b}\left(y_{2 p^{\prime}}, y_{2 p^{\prime}+1}\right), D_{q b}\left(y_{2 p^{\prime}+1}, y_{2 p^{\prime}}\right)\right\} \ngtr 0
\end{gathered}
$$

for some $p^{\prime} \in \mathbb{N} \cup\{0\}$, then

$$
\begin{aligned}
H_{d_{q b}}\left(S y_{2 p^{\prime}}, T y_{2 p^{\prime}+1}\right) & =H_{d_{q b}}\left(T y_{2 p^{\prime}+1}, S y_{2 p^{\prime}}\right) \\
& =D_{q b}\left(y_{2 p^{\prime}}, y_{2 p^{\prime}+1}\right) \\
& =D_{q b}\left(y_{2 p^{\prime}+1}, y_{2 p^{\prime}}\right)=0
\end{aligned}
$$

Clearly, if $D_{q b}\left(y_{2 p^{\prime}}, y_{2 p^{\prime}+1}\right)=0$, then $d_{q b}\left(y_{2 p^{\prime}}, y_{2 p^{\prime}+1}\right)=0$. Also $D_{q b}\left(y_{2 p^{\prime}+1}, y_{2 p^{\prime}}\right)=0$ implies $d_{q b}\left(y_{2 p^{\prime}+1}, y_{2 p^{\prime}}\right)=0$. So, $y_{2 p^{\prime}}=y_{2 p^{\prime}+1}$ and $y_{2 p^{\prime}} \in S y_{2 p^{\prime}}$. Now, $H_{d_{q b}}\left(S y_{2 p^{\prime}}, T y_{2 p^{\prime}+1}\right)=0$ implies $d_{q b}\left(y_{2 p^{\prime}+1}, T y_{2 p^{\prime}+1}\right)=0$ and $H_{d_{q b}}\left(T y_{2 p^{\prime}+1}, S y_{2 p^{\prime}}\right)=0$ implies $d_{q b}\left(T y_{2 p^{\prime}+1}, y_{2 p^{\prime}+1}\right)=0$. So, $y_{2 p^{\prime}+1} \in T y_{2 p^{\prime}+1}$ and $y_{2 p^{\prime}}$ is a common fixed point of $S$ and $T$. So the proof is completed in this case. Now, let

$$
\begin{gathered}
\max \left\{H_{d_{q b}}\left(S y_{2 p}, T y_{2 p+1}\right), H_{d_{q b}}\left(T y_{2 p+1}, S y_{2 p}\right),\right. \\
\left.D_{q b}\left(y_{2 p}, y_{2 p+1}\right), D_{q b}\left(y_{2 p+1}, y_{2 p}\right)\right\}>0,
\end{gathered}
$$

for all $p \in \mathbb{N} \cup\{0\}$. By Lemma 6, we have

$$
\begin{aligned}
& d_{q b}\left(y_{2 p}, y_{2 p+1}\right) \leq H_{d_{q b}}\left(T y_{2 p-1}, S y_{2 p}\right), \\
& d_{q b}\left(y_{2 p+1}, y_{2 p}\right) \leq H_{d_{q b}}\left(S y_{2 p}, T y_{2 p-1}\right),
\end{aligned}
$$

and

$$
\begin{aligned}
& d_{q b}\left(y_{2 p+1}, y_{2 p+2}\right) \leq H_{d_{q b}}\left(S y_{2 p}, T y_{2 p+1}\right), \\
& d_{q b}\left(y_{2 p+2}, y_{2 p+1}\right) \leq H_{d_{q b}}\left(T y_{2 p+1}, S y_{2 p}\right) .
\end{aligned}
$$

From (11), (F1) and using condition (5), we get

$$
\begin{aligned}
F & \left(s d_{q b}\left(y_{2 p+1}, y_{2 p+2}\right)\right) \leq F\left(s H_{d_{q b}}\left(S y_{2 p}, T y_{2 p+1}\right)\right) \\
& \leq \max \left\{F\left(s H_{d_{q b}}\left(S y_{2 p}, T y_{2 p+1}\right)\right),\right. \\
& \left.F\left(s H_{d_{q b}}\left(T y_{2 p+1}, S y_{2 p}\right)\right)\right\} \\
& \leq \min \left\{F\left(D_{q b}\left(y_{2 p}, y_{2 p+1}\right)\right), F\left(D_{q b}\left(y_{2 p+1}, y_{2 p}\right)\right)\right\} \\
& -\tau \leq F\left(D_{q b}\left(y_{2 p}, y_{2 p+1}\right)\right)-\tau,
\end{aligned}
$$

From (6), we have

$$
\begin{aligned}
& D_{q b}\left(y_{2 p}, y_{2 p+1}\right)=\max \left\{d_{q b}\left(y_{2 p}, y_{2 p+1}\right),\right. \\
& \frac{d_{q b}\left(y_{2 p}, S y_{2 p}\right) \cdot d_{q b}\left(y_{2 p+1}, T y_{2 p+1}\right)}{a+\max \left\{d_{q b}\left(y_{2 p}, y_{2 p+1}\right), d_{q b}\left(y_{2 p+1}, y_{2 p}\right)\right\}}, \\
& \left.d_{q b}\left(y_{2 p}, S y_{2 p}\right), d_{q b}\left(y_{2 p+1}, T y_{2 p+1}\right)\right\} \\
& =\max \left\{d_{q b}\left(y_{2 p}, y_{2 p+1}\right),\right. \\
& \frac{d_{q b}\left(y_{2 p}, y_{2 p+1}\right) \cdot d_{q b}\left(y_{2 p+1}, y_{2 p+2}\right)}{a+\max \left\{d_{q b}\left(y_{2 p}, y_{2 p+1}\right), d_{q b}\left(y_{2 p+1}, y_{2 p}\right)\right\}}, \\
& \left.d_{q b}\left(y_{2 p}, y_{2 p+1}\right), d_{q b}\left(y_{2 p+1}, y_{2 p+2}\right)\right\} \\
& \leq \max \left\{d_{q b}\left(y_{2 p}, y_{2 p+1}\right), d_{q b}\left(y_{2 p+1}, y_{2 p+2}\right)\right\} .
\end{aligned}
$$

If $\max \left\{d_{q b}\left(y_{2 p}, y_{2 p+1}\right), d_{q b}\left(y_{2 p+1}, y_{2 p+2}\right)\right\}=d_{q b}\left(y_{2 p+1}, y_{2 p+2}\right)$, then

$$
F\left(s d_{q b}\left(y_{2 p+1}, y_{2 p+2}\right)\right) \leq F\left(d_{q b}\left(y_{2 p+1}, y_{2 p+2}\right)\right)-\tau,
$$

which is a contradiction due to (F1) and $s \geq 1$. Therefore,

$$
F\left(s d_{q b}\left(y_{2 p+1}, y_{2 p+2}\right)\right) \leq F\left(d_{q b}\left(y_{2 p}, y_{2 p+1}\right)\right)-\tau,
$$

$$
\begin{aligned}
& F\left(s d_{q b}\left(y_{2 p+1}, y_{2 p+2}\right)\right) \\
& \quad \leq F\left(\max \left\{d_{q b}\left(y_{2 p}, y_{2 p+1}\right), d_{q b}\left(y_{2 p+1}, y_{2 p}\right)\right\}\right)
\end{aligned}
$$


From (11), (F1) and using condition (5), we get

$$
\begin{aligned}
& F\left(s d_{q b}\left(y_{2 p+2}, y_{2 p+1}\right)\right) \leq F\left(s H_{d_{q b}}\left(T y_{2 p+1}, S y_{2 p}\right)\right) \\
& \quad \leq \max \left\{F\left(s H_{d_{q b}}\left(S y_{2 p}, T y_{2 p+1}\right)\right),\right. \\
& \left.\quad F\left(s H_{d_{q b}}\left(T y_{2 p+1}, S y_{2 p}\right)\right)\right\} \\
& \quad \leq \min \left\{F\left(D_{q b}\left(y_{2 p}, y_{2 p+1}\right)\right), F\left(D_{q b}\left(y_{2 p+1}, y_{2 p}\right)\right)\right\} \\
& \quad-\tau \leq F\left(D_{q b}\left(y_{2 p}, y_{2 p+1}\right)\right)-\tau \\
& \quad=F\left(\max \left\{d_{q b}\left(y_{2 p}, y_{2 p+1}\right), d_{q b}\left(y_{2 p+1}, y_{2 p+2}\right)\right\}\right) \\
& \quad-\tau
\end{aligned}
$$

By using (15) and (F1), we get

$$
\begin{aligned}
& F\left(s d_{q b}\left(y_{2 p+2}, y_{2 p+1}\right)\right) \\
& \leq F\left(\max \left\{d_{q b}\left(y_{2 p}, y_{2 p+1}\right), \frac{1}{s} d_{q b}\left(y_{2 p}, y_{2 p+1}\right)\right\}\right) \\
&-\tau=F\left(d_{q b}\left(y_{2 p}, y_{2 p+1}\right)\right)-\tau \\
& \leq F\left(\max \left\{d_{q b}\left(y_{2 p}, y_{2 p+1}\right), d_{q b}\left(y_{2 p+1}, y_{2 p}\right)\right\}\right) \\
&-\tau . \\
& F\left(s d_{q b}\left(y_{2 p+2}, y_{2 p+1}\right)\right) \\
& \leq F\left(\max \left\{d_{q b}\left(y_{2 p}, y_{2 p+1}\right), d_{q b}\left(y_{2 p+1}, y_{2 p}\right)\right\}\right) \\
&-\tau .
\end{aligned}
$$

Combining (16) and (19), we get

$$
\begin{aligned}
& \max \left\{F\left(s d_{q b}\left(y_{2 p+2}, y_{2 p+1}\right)\right), F\left(s d_{q b}\left(y_{2 p+1}, y_{2 p+2}\right)\right)\right\} \\
& \quad \leq F\left(\max \left\{d_{q b}\left(y_{2 p}, y_{2 p+1}\right), d_{q b}\left(y_{2 p+1}, y_{2 p}\right)\right\}\right) \\
& \quad-\tau
\end{aligned}
$$

By using (10) and (5), we have

$$
\begin{aligned}
& F\left(s d_{q b}\left(y_{2 p}, y_{2 p+1}\right)\right) \leq F\left(s H_{d_{q b}}\left(T y_{2 p-1}, S y_{2 p}\right)\right) \\
& \quad \leq \max \left\{F\left(s H_{d_{q b}}\left(s y_{2 p}, T y_{2 p-1}\right)\right),\right. \\
& \left.\quad F\left(s H_{d_{q b}}\left(T y_{2 p-1}, S y_{2 p}\right)\right)\right\} \\
& \quad \leq \min \left\{F\left(D_{q b}\left(y_{2 p-1}, y_{2 p}\right)\right), F\left(D_{q b}\left(y_{2 p}, y_{2 p-1}\right)\right)\right\} \\
& \quad-\tau \leq F\left(D_{q b}\left(y_{2 p}, y_{2 p-1}\right)\right)-\tau .
\end{aligned}
$$

From (6), we have

$$
\begin{aligned}
& D_{q b}\left(y_{2 p}, y_{2 p-1}\right)=\max \left\{d_{q b}\left(y_{2 p}, y_{2 p-1}\right),\right. \\
& \frac{d_{q b}\left(y_{2 p}, y_{2 p+1}\right) \cdot d_{q b}\left(y_{2 p-1}, y_{2 p}\right)}{a+\max \left\{d_{q b}\left(y_{2 p}, y_{2 p-1}\right), d_{q b}\left(y_{2 p-1}, y_{2 p}\right)\right\}}, \\
& \left.d_{q b}\left(y_{2 p}, y_{2 p+1}\right), d_{q b}\left(y_{2 p-1}, y_{2 p}\right)\right\} \\
& \leq \max \left\{d_{q b}\left(y_{2 p}, y_{2 p-1}\right), d_{q b}\left(y_{2 p-1}, y_{2 p}\right),\right. \\
& \left.d_{q b}\left(y_{2 p}, y_{2 p+1}\right)\right\} .
\end{aligned}
$$

If $\max \left\{d_{q b}\left(y_{2 p}, y_{2 p-1}\right), d_{q b}\left(y_{2 p-1}, y_{2 p}\right), d_{q b}\left(y_{2 p}, y_{2 p+1}\right)\right\}=$ $d_{q b}\left(y_{2 p}, y_{2 p+1}\right)$, then we obtain

$$
F\left(s d_{q b}\left(y_{2 p}, y_{2 p+1}\right)\right) \leq F\left(d_{q b}\left(y_{2 p}, y_{2 p+1}\right)\right)-\tau,
$$

which is a contradiction due to (F1). Therefore,

$$
\begin{aligned}
& F\left(s d_{q b}\left(y_{2 p}, y_{2 p+1}\right)\right) \\
& \leq F\left(\max \left\{d_{q b}\left(y_{2 p-1}, y_{2 p}\right), d_{q b}\left(y_{2 p}, y_{2 p-1}\right)\right\}\right) \\
& \quad-\tau .
\end{aligned}
$$

By using (10) and (5), we have

$$
\begin{aligned}
& F\left(s d_{q b}\left(y_{2 p+1}, y_{2 p}\right)\right) \leq F\left(s H_{d_{q b}}\left(s y_{2 p}, T y_{2 p-1}\right)\right) \\
& \quad \leq F\left(D_{q b}\left(y_{2 p}, y_{2 p-1}\right)\right)-\tau \\
& \quad \leq F\left(\operatorname { m a x } \left\{d_{q b}\left(y_{2 p}, y_{2 p-1}\right), d_{q b}\left(y_{2 p-1}, y_{2 p}\right),\right.\right. \\
& \left.\left.\quad d_{q b}\left(y_{2 p}, y_{2 p+1}\right)\right\}\right)-\tau .
\end{aligned}
$$
From $\quad(24), \quad d_{q b}\left(y_{2 p}, y_{2 p+1}\right)$
$\max \left\{d_{q b}\left(y_{2 p-1}, y_{2 p}\right), d_{q b}\left(y_{2 p}, y_{2 p-1}\right)\right\}$, so

$$
\begin{aligned}
& F\left(s d_{q b}\left(y_{2 p+1}, y_{2 p}\right)\right) \\
& \quad \leq F\left(\max \left\{d_{q b}\left(y_{2 p}, y_{2 p-1}\right), d_{q b}\left(y_{2 p-1}, y_{2 p}\right)\right\}\right)
\end{aligned}
$$

$$
-\tau \text {. }
$$

Combining (24) and (26), we get

$$
\begin{gathered}
\max \left\{F\left(s d_{q b}\left(y_{2 p}, y_{2 p+1}\right)\right), F\left(s d_{q b}\left(y_{2 p+1}, y_{2 p}\right)\right)\right\} \\
\leq \max \left\{d_{q b}\left(y_{2 p}, y_{2 p-1}\right), d_{q b}\left(y_{2 p-1}, y_{2 p}\right)\right\}-\tau .
\end{gathered}
$$

Combining (20) and (27), we get

$$
\begin{array}{r}
\tau+F\left(s \max \left\{d_{q b}\left(y_{n}, y_{n+1}\right), d_{q b}\left(y_{n+1}, y_{n}\right)\right\}\right) \\
\leq F\left(\max \left\{d_{q b}\left(y_{n-1}, y_{n}\right), d_{q b}\left(y_{n}, y_{n-1}\right)\right\}\right)
\end{array}
$$


By Lemma $8,\left\{T S\left(y_{n}\right)\right\}$ is a Cauchy sequence in $\left(Y, d_{q b}\right)$. Since $\left(Y, d_{q b}\right)$ is a complete dislocated $b$-quasi-metric space, so there exists $u \in Y$ such that $\left\{T S\left(y_{n}\right)\right\} \longrightarrow u$; that is,

$$
\lim _{n \longrightarrow \infty} d_{q b}\left(y_{n}, u\right)=\lim _{n \longrightarrow \infty} d_{q b}\left(u, y_{n}\right)=0 .
$$

Now, suppose $d_{q b}(u, T u)>0$, and then $D_{q b}\left(y_{2 n}, u\right)>0$, so

$$
\begin{aligned}
& \max \left\{H_{d_{q b}}\left(S y_{2 n}, T u\right), H_{d_{q b}}\left(T u, S y_{2 n}\right), D_{q b}\left(y_{2 n}, u\right),\right. \\
& \left.D_{q b}\left(u, y_{2 n}\right)\right\}>0 .
\end{aligned}
$$

By using Lemma 6 and (5), we have

$$
\begin{aligned}
\tau+ & F\left(s d_{q b}\left(y_{2 n+1}, T u\right)\right) \leq \tau \\
& +\max \left\{F\left(s H_{d_{q b}}\left(s y_{2 n}, T u\right)\right),\right. \\
& \left.F\left(s H_{d_{q b}}\left(T u, S y_{2 n}\right)\right)\right\} \leq \min \left\{F\left(D_{q b}\left(y_{2 n}, u\right)\right),\right. \\
& \left.F\left(D_{q b}\left(u, y_{2 n}\right)\right)\right\} \leq F\left(D_{q b}\left(y_{2 n}, u\right)\right) .
\end{aligned}
$$

Since $F$ is strictly increasing, we have

$$
s d_{q b}\left(y_{2 n+1}, T u\right)<D_{q b}\left(y_{2 n}, u\right) .
$$

Taking $\lim _{n \rightarrow \infty}$ on both sides, we get

$$
\lim _{n \longrightarrow \infty} s d_{q b}\left(y_{2 n+1}, T u\right)<\lim _{n \longrightarrow \infty} D_{q b}\left(y_{2 n}, u\right)
$$

From (6)

$$
\begin{gathered}
D_{q b}\left(y_{2 n}, u\right)=\max \left\{d_{q b}\left(y_{2 n}, u\right),\right. \\
\frac{d_{q b}\left(y_{2 n}, y_{2 n+1}\right) \cdot d_{q b}(u, T u)}{a+\max \left\{d_{q b}\left(y_{2 n}, u\right), d_{q b}\left(u, y_{2 n}\right)\right\}}, \\
\left.d_{q b}\left(y_{2 n}, y_{2 n+1}\right), d_{q b}(u, T u)\right\} .
\end{gathered}
$$

Taking limit as $n \longrightarrow \infty$, and by using (29), we get

$$
\lim _{n \longrightarrow \infty} D_{q b}\left(y_{2 n}, u\right)=d_{q b}(u, T u) .
$$

Using inequality (35) in (33), we get

$$
\lim _{n \rightarrow \infty} s d_{q b}\left(y_{2 n+1}, T u\right)<d_{q b}(u, T u) .
$$

Now,

$$
d_{q b}(u, T u) \leq s d_{q b}\left(u, y_{2 n+1}\right)+s d_{q b}\left(y_{2 n+1}, T u\right) .
$$

Taking limit as $n \longrightarrow \infty$,

$$
\begin{aligned}
d_{q b}(u, T u) \leq & s \lim _{n \longrightarrow \infty} d_{q b}\left(u, y_{2 n+1}\right) \\
& +\lim _{n \longrightarrow \infty} s d_{q b}\left(y_{2 n+1}, T u\right) .
\end{aligned}
$$

Using inequalities (29) and (36) in (38), we get

$$
d_{q b}(u, T u)<d_{q b}(u, T u) .
$$

This is a contradiction, so $d_{q b}(u, T u)=0$. Now, suppose $d_{q b}(T u, u)>0$, and then there exists $n_{0} \in \mathbb{N}$ such that $d_{q b}\left(T u, y_{2 n+1}\right)>0$ for all $n \geq n_{0}$. By Lemma $6 d_{q b}\left(T u, y_{2 n+1}\right) \leq H_{d_{q b}}\left(T u, S y_{2 n}\right)$, so

$$
\begin{aligned}
& \max \left\{H_{d_{q b}}\left(S y_{2 n}, T u\right), H_{d_{q b}}\left(T u, S y_{2 n}\right), D_{q b}\left(y_{2 n}, u\right),\right. \\
& \left.D_{q b}\left(u, y_{2 n}\right)\right\}>0 .
\end{aligned}
$$

for all $n \geq n_{0}$. Following similar arguments as above, we get

$$
\lim _{n \longrightarrow \infty} s d_{q b}\left(T u, y_{2 n+1}\right)<d_{q b}(u, T u)=0 .
$$

Now,

$$
d_{q b}(T u, u) \leq s d_{q b}\left(T u, y_{2 n+1}\right)+s d_{q b}\left(y_{2 n+1}, u\right) .
$$

Taking limit as $n \longrightarrow \infty$, and using inequalities (29) and (41), we get

$$
d_{q b}(T u, u) \leq 0
$$

which is a contradiction, so $d_{q b}(T u, u)=0$. Hence $u \in T u$. Similarly by using (29), Lemma 6 , and the inequality

$$
\tau+d_{q b}\left(S u, y_{2 n+2}\right) \leq \tau+F\left(H_{d_{q b}}\left(S u, T y_{2 n+1}\right)\right)
$$

we can show that $d_{q b}(S u, u)=0$. Similarly, $d_{q b}(u, S u)=0$. Hence, the pair $(S, T)$ has a common fixed point $u$ in $\left(Y, d_{q b}\right)$. Now,

$$
d_{q b}(u, u) \leq d_{q b}(u, T u)+d_{q b}(T u, u) \leq 0 .
$$

This implies that $d_{q b}(u, u)=0$. Hence the proof is completed.

Now, let us introduce the following example.

Example 11. Let $Y=\{0\} \cup \mathbb{Q}^{+}$and $d_{q b}(x, y)=(x+2 y)^{2}$ if $x \neq y$, and $d_{q b}(x, y)=0$, if $x=y$. Then $\left(Y, d_{q b}\right)$ is a dislocated $b$-quasi-metric space with $s=2$. Define the mappings $S, T$ : $Y \longrightarrow P(Y)$ as follows:

$$
\begin{aligned}
& S(y) \\
& =\left\{\begin{array}{cc}
{\left[\frac{1}{4} y, \frac{2}{5} y\right] \cap \mathbb{Q}^{+},} & \text {for all } y \in\left\{0,7, \frac{7}{4}, \frac{7}{12}, \frac{7}{48}, \ldots\right\}, \\
{[y+1, y+4] \cap \mathbb{Q}^{+},} & \text {otherwise. }
\end{array}\right\} \\
& T(y) \\
& =\left\{\begin{array}{cc}
{\left[\frac{1}{3} y, \frac{3}{8} y\right] \cap \mathbb{Q}^{+},} & \text {for all } \left.y \in\left\{0,7, \frac{7}{4}, \frac{7}{12}, \frac{7}{48}, \ldots\right\},\right\} \\
{[y+3, y+6] \cap \mathbb{Q}^{+},} & \text {otherwise. }
\end{array}\right\}
\end{aligned}
$$

Case 1. If $\tau+\max \left\{F\left(s H_{d_{a b}}(S x, T y)\right), F\left(s H_{d_{a b}}(T x, S y)\right)\right\}=\tau+$ $F\left(s H_{d_{q b}}(S x, T y)\right) \leq \min \left\{F\left(D_{q b}(x, y)\right), F\left(D_{q b}(y, x)\right)\right\}$ holds. Define the function $F: R^{+} \longrightarrow R$ by $F(x)=\ln (x)$ for all 
$x \in R^{+}$and $\tau>0$. As $x, y \in Y, \tau=\ln (1.2)$ and by taking $y_{0}=$ 7 , we define the sequence $\left\{\operatorname{TS}\left(y_{n}\right)\right\}=\{7,7 / 4,7 / 12,7 / 48, \cdots\}$ in $Y$ generated by $y_{0}=7$. Also, $\left\{T S\left(y_{n}\right)\right\} \longrightarrow 0$. Now, if $x, y \in\left\{T S\left(y_{n}\right)\right\} \cup\{0\}$, we have

$$
\begin{aligned}
& s H_{d_{q b}}(S x, T y)=2 H_{d_{q b}}\left(\left[\frac{1}{4} x, \frac{2}{5} x\right],\left[\frac{1}{3} y, \frac{3}{8} y\right]\right)=2 \\
& \cdot \max \left[\left\{\sup _{a \in S x} d_{q b}\left(a,\left[\frac{1}{3} y, \frac{3}{8} y\right]\right),\right.\right. \\
& \left.\left.\sup _{b \in T y} d_{q b}\left(\left[\frac{1}{4} x, \frac{2}{5} x\right], b\right)\right\}\right]=2 \max \left\{d_{q b}\left(\frac{2 x}{5}, \frac{y}{3}\right),\right. \\
& \left.d_{q b}\left(\frac{x}{4}, \frac{3}{8} y\right)\right\}=2 \max \left\{\left(\frac{2 x}{5}+\frac{2 y}{3}\right)^{2},\left(\frac{x}{4}+\frac{3}{4}\right.\right. \\
& \left.\cdot y)^{2}\right\} .
\end{aligned}
$$

Also

$$
\begin{aligned}
& D_{q_{b}}(x, y)=\max \left\{d_{q b}(x, y),\right. \\
& \frac{d_{q b}(x,[x / 4,2 x / 5]) \cdot d_{q b}(y,[y / 3,3 y / 8])}{1+\max \left\{d_{q b}(x, y), d_{q b}(y, x)\right\}}, \\
& \left.d_{q b}\left(x,\left[\frac{x}{4}, \frac{2 x}{5}\right]\right), d_{q b}\left(y,\left[\frac{y}{3}, \frac{3 y}{8}\right]\right)\right\} \\
& =\max \left\{d_{q b}(x, y), \frac{d_{q b}(x, x / 4) \cdot d_{q b}(y, y / 3)}{1+\max \left\{d_{q b}(x, y), d_{q b}(y, x)\right\}},\right. \\
& \left.d_{q b}\left(x, \frac{x}{4}\right), d_{q b}\left(y, \frac{y}{3}\right)\right\}=\max \left\{(x+2 y)^{2},\right. \\
& \left.\quad \frac{(5 x y)^{2}}{4\left(1+(x+2 y)^{2}\right)},\left(\frac{3 x}{2}\right)^{2},\left(\frac{5 y}{3}\right)^{2}\right\}=(x+2 y)^{2} .
\end{aligned}
$$

Case (i). If $\max \left\{(2 x / 5+2 y / 3)^{2},(x / 4+(3 / 4) y)^{2}\right\}=(x / 4+$ $(3 / 4) y)^{2}$, and $\tau=\ln (1.2)$, then we have

$$
\begin{aligned}
3(x+3 y)^{2} & \leq 20(x+2 y)^{2} \\
\frac{6}{5}\left(\frac{x}{4}+\frac{3}{4} y\right)^{2} & \leq(x+2 y)^{2} \\
\ln (1.2)+\ln \left(\frac{x}{4}+\frac{3}{4} y\right)^{2} & \leq \ln (x+2 y)^{2} .
\end{aligned}
$$

This implies that

$$
\tau+F\left(s H_{d_{q b}}(S x, T y) \leq F\left(D_{q_{b}}(x, y)\right) .\right.
$$

Case (ii). Similarly, if $\max \left\{(2 x / 5+2 y / 3)^{2},(x / 4+(3 / 4) y)^{2}\right\}=$ $(2 x / 5+2 y / 3)^{2}$, and $\tau=\ln (1.2)$, then we have

$$
\begin{aligned}
48(3 x+5 y)^{2} & \leq 1125(x+2 y)^{2} \\
\frac{6}{5}\left(\frac{2 x}{5}+\frac{2 y}{3}\right)^{2} & \leq(x+2 y)^{2} \\
\ln (1.2)+\ln \left(\frac{2 x}{5}+\frac{2 y}{3}\right)^{2} & \leq \ln (x+2 y)^{2} .
\end{aligned}
$$

Hence,

$$
\tau+F\left(s H_{d_{q b}}(S x, T y) \leq F\left(D_{q_{b}}(x, y)\right) .\right.
$$

Case 2. If $\max \left\{\tau+F\left(s H_{d_{q b}}(S x, T y)\right), \tau+F\left(s H_{d_{q b}}(T x, S y)\right)\right\}=$ $\tau+F\left(s H_{d_{q b}}(T x, S y)\right)$ holds.

$$
\begin{aligned}
& s H_{d_{q b}}(T x, S y)=2 \max \left[\left\{\sup _{b \in T x} d_{q b}(b, S y),\right.\right. \\
& \left.\left.\sup _{a \in S y} d_{q b}(T x, a)\right\}\right]=2 \\
& \cdot \max \left[\left\{\sup _{b \in T x} d_{q b}\left(b,\left[\frac{1}{4} y, \frac{2}{5} y\right]\right),\right.\right. \\
& \left.\left.\sup _{a \in S y} d_{q b}\left(\left[\frac{1}{3} x, \frac{3}{8} x\right], a\right)\right\}\right]=2 \max \left\{d_{q b}\left(\frac{3 x}{8}, \frac{y}{4}\right),\right. \\
& \left.d_{q b}\left(\frac{x}{3}, \frac{2 y}{5}\right)\right\}=2 \max \left\{\left(\frac{3 x}{8}+\frac{2 y}{4}\right)^{2},\left(\frac{x}{3}\right.\right. \\
& \left.\left.+\frac{4 y}{5}\right)^{2}\right\},
\end{aligned}
$$

where

$$
\begin{aligned}
& D_{q_{b}}(y, x)=\max \left\{d_{q b}(y, x),\right. \\
& \frac{d_{q b}(x,[x / 4,2 x / 5]) \cdot d_{q b}(y,[y / 3,3 y / 8])}{1+\max \left\{d_{q b}(x, y), d_{q b}(y, x)\right\}}, \\
& \left.d_{q b}\left(x,\left[\frac{x}{4}, \frac{2 x}{5}\right]\right), d_{q b}\left(y,\left[\frac{y}{3}, \frac{3 y}{8}\right]\right)\right\} \\
& =\max \left\{d_{q b}(y, x),\right. \\
& \frac{d_{q b}(x, x / 4) \cdot d_{q b}(y, y / 3)}{1+\max \left\{d_{q b}(x, y), d_{q b}(y, x)\right\}}, d_{q b}\left(x, \frac{x}{4}\right),
\end{aligned}
$$

$\left.d_{q b}\left(y, \frac{y}{3}\right)\right\}$ 


$$
\begin{aligned}
& D_{q_{b}}(y, x)=\max \left\{(y+2 x)^{2}, \frac{(5 x y)^{2}}{4\left(1+(y+2 x)^{2}\right)},\right. \\
& \left.\left(\frac{3 x}{2}\right)^{2},\left(\frac{5 y}{3}\right)^{2}\right\}=(y+2 x)^{2} .
\end{aligned}
$$

Case (i). If $\max \left\{(3 x / 8+2 y / 4)^{2},(x / 3+4 y / 5)^{2}\right\}=(x / 3+4 y / 5)^{2}$, and $\tau=\ln (1.2)$, then we have

$$
\begin{aligned}
12(5 x+12 y)^{2} & \leq 1125(y+2 x)^{2} \\
\frac{6}{5}\left(\frac{x}{3}+\frac{4 y}{5}\right)^{2} & \leq(y+2 x)^{2} \\
\ln (1.2)+\ln \left(\left(\frac{x}{3}+\frac{4 y}{5}\right)^{2}\right. & \leq \ln (y+2 x)^{2},
\end{aligned}
$$

so

$$
\tau+F\left(s H_{d_{q b}}(T x, S y) \leq F\left(D_{q_{b}}(y, x)\right) .\right.
$$

Case (ii). Similarly, if $\max \left\{(3 x / 8+2 y / 4)^{2},(x / 3+4 y / 5)^{2}\right\}=$ $(3 x / 8+2 y / 4)^{2}$, and $\tau=\ln (1.2)$, then we have

$$
\begin{aligned}
12(3 x+4 y)^{2} & \leq 320(y+2 x)^{2} \\
\frac{6}{5}\left(\frac{3 x}{8}+\frac{2 y}{4}\right)^{2} & \leq(y+2 x)^{2} \\
\ln (1.2)+\ln \left(\frac{3 x}{8}+\frac{2 y}{4}\right)^{2} & \leq \ln (y+2 x)^{2} .
\end{aligned}
$$

Hence,

$$
\tau+F\left(s H_{d_{q b}}(T x, S y) \leq F\left(D_{q_{b}}(y, x)\right) .\right.
$$

Now, if $x, y \notin\left\{\operatorname{TS}\left(y_{n}\right)\right\}$, then the contraction does not hold. Hence all the hypotheses of Theorem 10 are satisfied so $S$ and $T$ have a common fixed point.

If we take $S=T$ in Theorem 10, then we obtain the following theorem.

Theorem 12. Let $\left(Y, d_{q b}\right)$ be a complete dislocated b-quasimetric space with $s \geq 1$ and $S: Y \longrightarrow P(Y)$ be a multivalued mapping such that for every two consecutive points $x, y$ belonging to the range of an iterative sequence $\left\{S\left(y_{n}\right)\right\}$ with $D_{q_{b}}(x, y)>0, F \in \mathscr{F}_{s}, \tau, a>0$

$$
\tau+F\left(s H_{q_{b}}(S x, S y)\right) \leq F\left(D_{q_{b}}(x, y)\right),
$$

where

$$
\begin{gathered}
D_{q_{b}}(x, y)=\max \left\{d_{q b}(x, y), \frac{d_{q b}(x, S x) \cdot d_{q b}(y, S y)}{a+d_{q b}(x, y)},\right. \\
\left.d_{q b}(x, S x), d_{q b}(y, S y)\right\} .
\end{gathered}
$$

Then $\left\{S\left(y_{n}\right)\right\} \longrightarrow u \in Y$. Moreover, if (59) also holds for $u$, then $S$ has a fixed point $u$ in $Y$ and $d_{q b}(u, u)=0$.

Remark 13. By setting the different values of $D_{q b}(x, y)$ in (6), we can obtain different results on multivalued $F$-contractions as corollaries of Theorem 10.

\section{3. $F \rho_{s}^{*}$-Khan Type Contraction in Quasi b-Metric Spaces}

Piri et al. [42] extended the results of Khan [43] and Fisher [44] by introducing a new general contractive condition with rational expressions. Recently, Piri et al. [30] improved some fixed point results of $F_{k}$-Khan type self-mapping on complete metric spaces. In this section, we introduce a new type of contraction satisfying an inequality of rational expressions and prove a new fixed point theorem concerning this type of contraction. Our result is real generalization of Khan fixed point theorem; we introduced $F \rho_{s}^{*}$-Khan type multivalued for two mappings in $b$-quasi-metric space. We start this section with the following definitions.

Definition 14. Let $Y$ be a nonempty set, $s \geq 1$, and $\rho_{s}$ : $X \times X \longrightarrow[0,+\infty)$ be a mapping such that $\rho_{s}(x, y) \geq$ $s$ and $\rho_{s}(y, x) \geq s$, implying $x=y$. Let $M \subseteq Y$ define $\rho_{s}^{*}(x, M)=\inf \left\{\rho_{s}(x, a), a \in M\right\}$ and $\rho_{s}^{*}(M, y)=$ $\inf \left\{\rho_{s}(b, y), b \in M\right\}$. Let $S, T: Y \longrightarrow P(Y)$ be the multivalued mappings; then the pair $(S, T)$ is said to be $\rho_{s}^{*}$-Alt multivalued mapping; if $x \in Y$, then

(a) $\rho_{s}^{*}(x, S x) \geq s$,

$$
q_{b}(x, S x)=q_{b}(x, y)
$$

and $q_{b}(S x, x)=q_{b}(y, x)$ implies $\rho_{s}^{*}(S y, y) \geq s$,

(b) $\rho_{s}^{*}(S x, x) \geq s$,

$$
\begin{aligned}
q_{b}(x, T x) & =q_{b}(x, y) \\
\text { and } q_{b}(T x, x) & =q_{b}(y, x) \quad \text { implies } \rho_{s}^{*}(y, S y) \geq s .
\end{aligned}
$$

Definition 15 (see [30]). Let $(X, d)$ be a metric space. A mapping $T: X \longrightarrow X$ is said to be $F$-Khan type contraction if there exists $\tau \in(0, \infty)$ and $F \in \mathscr{F}_{k}$ such that

$$
\begin{aligned}
\tau+ & F(d(T x, T y)) \\
& \leq F\left(\frac{d(x, T x) \cdot d(x, T y)+d(y, T y) \cdot d(y, T x)}{\max \{d(x, T y), d(y, T x)\}}\right),
\end{aligned}
$$

for all $x, y \in X$, and if $\max \{d(x, T y), d(y, T x)\} \neq 0$, then $T x \neq T y$ and if $\max \{d(x, T y), d(y, T x)\}=0$, then $T x=T y$.

Definition 16. Let $\left(Y, q_{b}, s\right)$ be a $b$-quasi-metric space and $(S, T)$ be a pair of $\rho_{s}^{*}$ multivalued mappings. Then $(S, T)$ is called $F \rho_{s}^{*}$ Khan type contraction, if there exists $F \in$ $\mathscr{F}_{S}$ and $\tau>0$ such that for every two consecutive points $x, y$ belonging to the range of an iterative sequence $\left\{T S\left(y_{n}\right)\right\}$ with $\rho_{s}^{*}(S y, y) \geq s, \rho_{s}^{*}(x, S x) \geq s$, and 
$\max \left\{H_{q_{b}}(S x, T y), H_{q_{b}}(T y, S x), q_{b}(x, y), q_{b}(y, x)\right\}>0$, we have

$$
\begin{aligned}
\tau+ & \max \left\{F\left(s H_{q_{b}}(S x, T y)\right), F\left(s H_{q_{b}}(T y, S x)\right.\right. \\
& \leq \min \left\{F\left(Q_{b}(x, y)\right), F\left(Q_{b}(y, x)\right)\right\},
\end{aligned}
$$

where

$$
\begin{aligned}
& Q_{b}(x, y) \\
& \quad=\frac{q_{b}(x, S x) q_{b}(x, T y)+q_{b}(y, T y) q_{b}(y, S x)}{\max \left\{q_{b}(x, T y), q_{b}(y, S x)\right\}} .
\end{aligned}
$$

Theorem 17. Let $\left(Y, q_{b}, s\right)$ be a complete b-quasi-metric space with $s \geq 1$. Let $\rho_{s}: Y \times Y \longrightarrow[0,+\infty)$ and $(S, T)$ be a pair of $F \rho_{s}^{*}$ Khan type contractions and the set $G(S)=\{x$ : $\left.\rho_{s}^{*}(x, S x) \geq s\right\}$ is closed and contained $y_{0}$. Then $\left\{T S\left(y_{n}\right)\right\} \longrightarrow$ $u \in Y$. Also, if (63) holds for each $x, y \in\{u\}$, then $S$ and $T$ have a common fixed point $u$ in $Y$ and $q_{b}(u, u)=0$.

Proof. As $y_{0}$ is an arbitrary element of $G(S)$, from condition of the theorem $\rho_{s}^{*}\left(y_{0}, S y_{0}\right) \geq s$. Let $\left\{T S\left(y_{n}\right)\right\}$ be the iterative sequence in $Y$ generated by a point $y_{0} \in Y$. Let $y_{2 p^{\prime}}, y_{2 p^{\prime}+1}$ be elements of this sequence. Clearly, if

$$
\begin{gathered}
\max \left\{H_{q_{b}}\left(S y_{2 p^{\prime}}, T y_{2 p^{\prime}+1}\right), H_{q_{b}}\left(T y_{2 p^{\prime}+1}, S y_{2 p^{\prime}}\right),\right. \\
\left.q_{b}\left(y_{2 p^{\prime}}, y_{2 p^{\prime}+1}\right), q_{b}\left(y_{2 p^{\prime}+1}, y_{2 p^{\prime}}\right)\right\} \ngtr 0,
\end{gathered}
$$

for some $p^{\prime} \in \mathbb{N} \cup\{0\}$, then

$$
\begin{aligned}
H_{q_{b}}\left(S y_{2 p^{\prime}}, T y_{2 p^{\prime}+1}\right) & =H_{q_{b}}\left(T y_{2 p^{\prime}+1}, S y_{2 p^{\prime}}\right) \\
& =q_{b}\left(y_{2 p^{\prime}}, y_{2 p^{\prime}+1}\right) \\
& =q_{b}\left(y_{2 p^{\prime}+1}, y_{2 p^{\prime}}\right)=0 .
\end{aligned}
$$

As $q_{b}\left(y_{2 p^{\prime}}, y_{2 p^{\prime}+1}\right)=q_{b}\left(y_{2 p^{\prime}+1}, y_{2 p^{\prime}}\right)=0$, so $y_{2 p^{\prime}}=y_{2 p^{\prime}+1}$ and $y_{2 p^{\prime}} \in S y_{2 p^{\prime}}$. Now, $H_{q b}\left(S y_{2 p^{\prime}}, T y_{2 p^{\prime}+1}\right)=0$ implies $q_{b}\left(y_{2 p^{\prime}+1}, T y_{2 p^{\prime}+1}\right)=0$ and $H_{q_{b}}\left(T y_{2 p^{\prime}+1}, S y_{2 p^{\prime}}\right)=0$ implies $q_{b}\left(T y_{2 p^{\prime}+1}, y_{2 p^{\prime}+1}\right)=0$. So, $y_{2 p^{\prime}+1} \in T y_{2 p^{\prime}+1}$ and $y_{2 p^{\prime}}$ is a common fixed point of $S$ and $T$. So the proof is done. In order to find common fixed point of both $S$ and $T$, when

$$
\begin{gathered}
\max \left\{H_{q_{b}}\left(S y_{2 p}, T y_{2 p+1}\right), H_{q_{b}}\left(T y_{2 p+1}, S y_{2 p}\right),\right. \\
\left.q_{b}\left(y_{2 p}, y_{2 p+1}\right), q_{b}\left(y_{2 p+1}, y_{2 p}\right)\right\}>0
\end{gathered}
$$

for all $p \in\{0\} \cup \mathbb{N}$. Since $\rho_{s}^{*}\left(y_{0}, S y_{0}\right) \geq s, q_{b}\left(y_{0}, S y_{0}\right)=$ $q_{b}\left(y_{0}, y_{1}\right)$ and $q_{b}\left(S y_{0}, y_{0}\right)=q_{b}\left(y_{1}, y_{0}\right)$. As $(S, T)$ is $\rho_{s}^{*}$ multivalued mapping, $\rho_{s}^{*}\left(S y_{1}, y_{1}\right) \geq s$. Now, $\rho_{s}^{*}\left(S y_{1}, y_{1}\right) \geq$ $s, q_{b}\left(y_{1}, T y_{1}\right)=q_{b}\left(y_{1}, y_{2}\right)$ and $q_{b}\left(T y_{1}, y_{1}\right)=q_{b}\left(y_{2}, y_{1}\right)$ implies that $\rho_{s}^{*}\left(y_{2}, S y_{2}\right) \geq s$. By induction we deduce that $\rho_{s}^{*}\left(y_{2 p}, S y_{2 p}\right) \geq s$ and $\rho_{s}^{*}\left(S y_{2 p+1}, y_{2 p+1}\right) \geq s$, for all $p=$ $0,1,2, \cdots$. Now, by Lemma 6 , we have

$$
\begin{aligned}
& q_{b}\left(y_{2 p}, y_{2 p+1}\right) \leq H_{q_{b}}\left(T y_{2 p-1}, S y_{2 p}\right), \\
& q_{b}\left(y_{2 p+1}, y_{2 p}\right) \leq H_{q_{b}}\left(S y_{2 p}, T y_{2 p-1}\right)
\end{aligned}
$$

and

$$
\begin{aligned}
& q_{b}\left(y_{2 p+1}, y_{2 p+2}\right) \leq H_{q_{b}}\left(S y_{2 p}, T y_{2 p+1}\right), \\
& q_{b}\left(y_{2 p+2}, y_{2 p+1}\right) \leq H_{q_{b}}\left(T y_{2 p+1}, S y_{2 p}\right) .
\end{aligned}
$$

As $s \geq 1$, then (69) implies

$$
\begin{aligned}
& F\left(s q_{b}\left(y_{2 p+1}, y_{2 p+2}\right)\right) \leq F\left(s H_{q_{b}}\left(s y_{2 p}, T y_{2 p+1}\right)\right) \\
& \quad \leq \max \left\{F\left(s H_{q_{b}}\left(s y_{2 p}, T y_{2 p+1}\right)\right),\right. \\
& \left.\quad F\left(s H_{q_{b}}\left(T y_{2 p+1}, S y_{2 p}\right)\right)\right\} .
\end{aligned}
$$

As $y_{2 p}, y_{2 p+1} \in\left\{T S\left(y_{n}\right)\right\}, \rho_{s}^{*}\left(y_{2 p}, S y_{2 p}\right) \geq s$ and $\rho_{s}^{*}\left(S y_{2 p+1}, y_{2 p+1}\right) \geq s$, then by using the condition (63), we get

$$
\begin{aligned}
& F\left(s q_{b}\left(y_{2 p+1}, y_{2 p+2}\right)\right) \\
& \leq \min \left\{F\left(Q_{b}\left(y_{2 p}, y_{2 p+1}\right)\right), F\left(Q_{b}\left(y_{2 p+1}, y_{2 p}\right)\right)\right\} \\
& \quad-\tau \leq F\left(Q_{b}\left(y_{2 p}, y_{2 p+1}\right)\right)-\tau .
\end{aligned}
$$

From (64), we get

$$
\begin{aligned}
Q_{b}\left(y_{2 p}, y_{2 p+1}\right) & =\frac{q_{b}\left(y_{2 p}, S y_{2 p}\right) q_{b}\left(y_{2 p}, T y_{2 p+1}\right)+q_{b}\left(y_{2 p+1}, T y_{2 p+1}\right) q_{b}\left(y_{2 p+1}, S y_{2 p}\right)}{\max \left\{q_{b}\left(y_{2 p}, T y_{2 p+1}\right), q_{b}\left(S y_{2 p}, y_{2 p+1}\right)\right\}} \\
& =\frac{q_{b}\left(y_{2 p}, y_{2 p+1}\right) \cdot q_{b}\left(y_{2 p}, T y_{2 p+1}\right)+q_{b}\left(y_{2 p+1}, y_{2 p+2}\right) \times 0}{\max \left\{q_{b}\left(y_{2 p}, T y_{2 p+1}\right), 0\right\}}=q_{b}\left(y_{2 p}, y_{2 p+1}\right) .
\end{aligned}
$$

Therefore,

$$
F\left(s q_{b}\left(y_{2 p+1}, y_{2 p+2}\right) \leq F\left(q_{b}\left(y_{2 p}, y_{2 p+1}\right)\right)-\tau\right.
$$

and this implies

$$
\begin{aligned}
& F\left(s q_{b}\left(y_{2 p+1}, y_{2 p+2}\right)\right) \\
& \quad \leq F\left(\max \left\{q_{b}\left(y_{2 p}, y_{2 p+1}\right), q_{b}\left(y_{2 p+1}, y_{2 p}\right)\right\}\right)-\tau .
\end{aligned}
$$


As $s \geq 1$, then (69) implies

$$
\begin{aligned}
& F\left(s q_{b}\left(y_{2 p+2}, y_{2 p+1}\right)\right) \leq F\left(s H_{q_{b}}\left(T y_{2 p+1}, S y_{2 p}\right)\right) \\
& \quad \leq \max \left\{F\left(s H_{q_{b}}\left(T y_{2 p+1}, S y_{2 p}\right)\right)\right. \\
& \left.\quad F\left(s H_{q_{b}}\left(S y_{2 p}, T y_{2 p+1}\right)\right)\right\}
\end{aligned}
$$

As $y_{2 p+1}, y_{2 p} \in\left\{T S\left(y_{n}\right)\right\}, \rho_{s}^{*}\left(S y_{2 p+1}, y_{2 p+1}\right) \geq s$ and $\rho_{s}^{*}\left(y_{2 p}, S y_{2 p}\right) \geq s$, then using condition (63), we get

$$
\begin{aligned}
F( & \left.s q_{b}\left(y_{2 p+2}, y_{2 p+1}\right)\right) \\
\leq & \min \left\{F\left(Q_{b}\left(y_{2 p}, y_{2 p+1}\right)\right), F\left(Q_{b}\left(y_{2 p+1}, y_{2 p}\right)\right)\right\} \\
& \quad-\tau \leq F\left(Q_{b}\left(y_{2 p}, y_{2 p+1}\right)\right)-\tau \\
= & F\left(q_{b}\left(y_{2 p}, y_{2 p+1}\right)\right)-\tau .
\end{aligned}
$$

Therefore,

$$
\begin{aligned}
& F\left(s q_{b}\left(y_{2 p+2}, y_{2 p+1}\right)\right) \\
& \quad \leq F\left(\max \left\{q_{b}\left(y_{2 p}, y_{2 p+1}\right), q_{b}\left(y_{2 p+1}, y_{2 p}\right)\right\}\right)-\tau .
\end{aligned}
$$

Combining (74) and (77), we get

$$
\begin{gathered}
\max \left\{F\left(s q_{b}\left(y_{2 p+1}, y_{2 p+2}\right)\right), F\left(s q_{b}\left(y_{2 p+2}, y_{2 p+1}\right)\right)\right\} \\
\leq F\left(\max \left\{q_{b}\left(y_{2 p}, y_{2 p+1}\right), q_{b}\left(y_{2 p+1}, y_{2 p}\right)\right\}\right)-\tau .
\end{gathered}
$$

As $s \geq 1$, then (68) implies

$$
\begin{aligned}
& F\left(s q_{b}\left(y_{2 p}, y_{2 p+1}\right)\right) \leq\left(s H_{q_{b}}\left(T y_{2 p-1}, S y_{2 p}\right)\right) \\
& \quad \leq \max \left\{F\left(s H_{q_{b}}\left(S y_{2 p}, T y_{2 p-1}\right)\right),\right. \\
& \left.\quad F\left(s H_{q_{b}}\left(T y_{2 p-1}, S y_{2 p}\right)\right)\right\}
\end{aligned}
$$

As $y_{2 p}, y_{2 p-1} \in\left\{T S\left(y_{n}\right)\right\}, \rho_{s}^{*}\left(y_{2 p}, S y_{2 p}\right) \geq s$ and $\rho_{s}^{*}\left(S y_{2 p-1}, y_{2 p-1}\right) \geq s$, then by using condition (63), we get

$$
\begin{aligned}
F\left(s q_{b}\left(y_{2 p}, y_{2 p+1}\right)\right) & \leq \min \left\{F\left(Q_{b}\left(y_{2 p}, y_{2 p-1}\right)\right), F\left(Q_{b}\left(y_{2 p-1}, y_{2 p}\right)\right)\right\}-\tau \leq F\left(Q_{b}\left(y_{2 p}, y_{2 p-1}\right)\right)-\tau \\
F\left(s q_{b}\left(y_{2 p}, y_{2 p+1}\right)\right) & \leq F\left(\frac{q_{b}\left(y_{2 p}, S y_{2 p}\right) \cdot q_{b}\left(y_{2 p}, T y_{2 p-1}\right)+q_{b}\left(y_{2 p-1}, T y_{2 p-1}\right) \cdot q_{b}\left(y_{2 p-1}, S y_{2 p}\right)}{\max \left\{q_{b}\left(y_{2 p}, T y_{2 p-1}\right), q_{b}\left(y_{2 p-1}, S y_{2 p}\right)\right\}}\right)-\tau \\
& \leq F\left(\frac{q_{b}\left(y_{2 p}, y_{2 p+1}\right) \cdot q_{b}\left(y_{2 p}, y_{2 p}\right)+q_{b}\left(y_{2 p-1}, y_{2 p}\right) \cdot q_{b}\left(y_{2 p-1}, S y_{2 p}\right)}{\max \left\{0, q_{b}\left(y_{2 p-1}, S y_{2 p}\right)\right\}}\right)-\tau \\
& \leq F\left(q_{b}\left(y_{2 p-1}, y_{2 p}\right)\right)-\tau .
\end{aligned}
$$

Therefore,

$$
\begin{aligned}
& F\left(s q_{b}\left(y_{2 p}, y_{2 p+1}\right)\right) \\
& \quad \leq F\left(\max \left\{q_{b}\left(y_{2 p-1}, y_{2 p}\right), q_{b}\left(y_{2 p}, y_{2 p-1}\right)\right\}\right)-\tau .
\end{aligned}
$$

Similarly, by using (63), (64), and (68), we get

$$
\begin{aligned}
& F\left(s q_{b}\left(y_{2 p+1}, y_{2 p}\right)\right) \\
& \quad \leq F\left(\max \left\{q_{b}\left(y_{2 p-1}, y_{2 p}\right), q_{b}\left(y_{2 p}, y_{2 p-1}\right)\right\}\right)-\tau .
\end{aligned}
$$

Combining (81) and (82), we get

$$
\begin{aligned}
\tau+ & F\left(s \max \left\{q_{b}\left(y_{2 p}, y_{2 p+1}\right)\right) F\left(q_{b}\left(y_{2 p+1}, y_{2 p}\right)\right)\right. \\
& \leq F\left(\max \left\{q_{b}\left(y_{2 p-1}, y_{2 p}\right), q_{b}\left(y_{2 p}, y_{2 p-1}\right)\right\}\right) .
\end{aligned}
$$

Combining (78) and (83), we get

$$
\begin{aligned}
\tau+ & F\left(s \max \left\{q_{b}\left(y_{n}, y_{n+1}\right), q_{b}\left(y_{n+1}, y_{n}\right)\right\}\right) \\
& \leq F\left(\max \left\{q_{b}\left(y_{n-1}, y_{n}\right), q_{b}\left(y_{n}, y_{n-1}\right)\right\}\right) .
\end{aligned}
$$

By Lemma $8, \quad\left\{T S\left(y_{n}\right)\right\}$ is a Cauchy sequence in $\left(Y, q_{b}\right) \cdot \rho_{s}^{*}\left(y_{2 p}, S y_{2 p}\right) \geq s$ for all $p \in \mathbb{N}$. So $\left\{y_{2 p}\right\}$ is a subsequence of $\left\{T S\left(y_{n}\right)\right\}$ contained in $G(S)$. As $G(S)$ is closed, there exists $u \in G(S)$ such that $\left\{y_{2 p}\right\} \longrightarrow u$, that is,

$$
\lim _{n \longrightarrow \infty} q_{b}\left(y_{n}, u\right)=\lim _{n \longrightarrow \infty} q_{b}\left(u, y_{n}\right)=0 \text {. }
$$

Also

$$
\rho_{s}^{*}(u, S u) \geq s .
$$

Now, we show that $u$ is a fixed point for $S$. We claim that $q_{b}(S u, u)=q_{b}(u, S u)=0$. On the contrary, we assume that $q_{b}(u, S u)>0$. Now

$$
q_{b}(u, S u) \leq s\left(q_{b}\left(u, y_{2 n}\right)+q_{b}\left(y_{2 n}, S u\right)\right) .
$$

So, there exists $n_{0} \in \mathbb{N}$ such that $q_{b}\left(y_{2 n}, S u\right)>0$ for all $n \geq n_{0}$. By Lemma 6, we have $0<q_{b}\left(y_{2 n}, S u\right) \leq H_{q_{b}}\left(T y_{2 n-1}, S u\right)$ for all $n \geq n_{0}$, so

$$
\begin{aligned}
& \max \left\{H_{q_{b}}\left(T y_{2 n-1}, S u\right), H_{q_{b}}\left(S u, T y_{2 n-1}\right), q_{b}\left(u, y_{2 n-1}\right),\right. \\
& \left.\quad q_{b}\left(y_{2 n-1}, u\right)\right\}>0,
\end{aligned}
$$


for all $n \geq n_{0}$. By Lemma 6 , and $s \geq 1$, we get

$$
\begin{aligned}
\tau+ & F\left(s q_{b}\left(y_{2 n}, S u\right)\right) \\
\leq & \tau \\
& +F\left(s \max \left\{H_{q_{b}}\left(T y_{2 n-1}, S u\right), H_{q_{b}}\left(S u, T y_{2 n-1}\right)\right\}\right) .
\end{aligned}
$$

Now, $\rho_{s}^{*}(u, S u) \geq s$ and $\rho_{s}^{*}\left(S y_{2 n-1}, y_{2 n-1}\right) \geq s$, and then by (64), we get

$$
\tau+F\left(s q_{b}\left(y_{2 n}, S u\right)\right) \leq F\left(Q_{b}\left(y_{2 n-1}, u\right)\right) .
$$

Since $F$ is strictly increasing, we have

$$
s q_{b}\left(y_{2 n}, S u\right)<Q_{b}\left(y_{2 n-1}, u\right) .
$$

Taking limit as $n \longrightarrow \infty$, on both sides of inequality (91), we get

$$
\lim _{n \longrightarrow \infty} s q_{b}\left(y_{2 n}, S u\right)<\lim _{n \longrightarrow \infty} Q_{b}\left(y_{2 n-1}, u\right)
$$

Since $q_{b}\left(u, T y_{2 n-1}\right) \leq q_{b}\left(u, y_{2 n}\right)$, taking limit as $n \longrightarrow \infty$, on both sides, we get

$$
\lim _{n \rightarrow \infty} q_{b}\left(u, T y_{2 n-1}\right)=0
$$

By (64), we have

$$
\begin{aligned}
& Q_{b}\left(y_{2 n-1}, u\right) \\
& =\frac{q_{b}\left(y_{2 n-1}, T y_{2 n-1}\right) q_{b}\left(y_{2 n-1}, S u\right)+q_{b}(u, S u) q_{b}\left(u, T y_{2 n-1}\right)}{\max \left\{q_{b}\left(y_{2 n-1}, S u\right), q_{b}\left(u, T y_{2 n-1}\right)\right\}}
\end{aligned}
$$

Taking limit as $n \longrightarrow \infty$ and using inequality (93), we have

$$
\lim _{n \rightarrow \infty} Q_{b}\left(y_{2 n-1}, u\right)=\lim _{n \longrightarrow \infty} q_{b}\left(y_{2 n-1}, y_{2 n}\right)=0 \text {. }
$$

Now, inequality (92) implies

$$
\lim _{n \rightarrow \infty} s q_{b}\left(y_{2 n}, S u\right)<0 .
$$

Taking limit as $n \longrightarrow \infty$ on both sides of inequality (87) and using the above inequality, we have

$$
q_{b}(u, S u)<0 .
$$

So our assumption is wrong and $q_{b}(u, S u)=0$. Now assume that $q_{b}(S u, u)>0$, and then there exists $n_{1} \in \mathbb{N}$ such that $q_{b}\left(S u, y_{2 n}\right)>0$ for all $n \geq n_{1}$. By Lemma $6 q_{b}\left(S u, y_{2 n}\right) \leq$ $H_{q_{b}}\left(S u, T y_{2 n-1}\right)$, so

$$
\begin{aligned}
& \max \left\{H_{q_{b}}\left(T y_{2 n-1}, S u\right), H_{q_{b}}\left(S u, T y_{2 n-1}\right), q_{b}\left(u, y_{2 n-1}\right)\right. \\
& \left.\quad q_{b}\left(y_{2 n-1}, u\right)\right\}>0
\end{aligned}
$$

for all $n \geq n_{1}$. Following similar arguments as above, we get

$$
\lim _{n \rightarrow \infty} s q_{b}\left(S u, y_{2 n}\right)<0 .
$$

Now,

$$
q_{b}(S u, u) \leq s q_{b}\left(S u, y_{2 n}\right)+s q_{b}\left(y_{2 n}, u\right)
$$

Taking limit as $n \longrightarrow \infty$, on both sides of inequality (100) and using (85) and (99), we get

$$
q_{b}(S u, u)<0
$$

which is a contradiction, so $q_{b}(S u, u)=0$. Hence $u \in S u$. As $\rho_{s}^{*}(u, S u) \geq s$ and $q_{b}(u, S u)=q_{b}(S u, u)=q_{b}(0,0)$, then Definition 14 implies

$$
\rho_{s}^{*}(S u, u) \geq s .
$$

Now, we show that $u$ is a fixed point for $T$. We claim that $q_{b}(u, T u)=0$. On the contrary, we assume that $q_{b}(u, T u)>0$, and then there exists $n_{2} \in \mathbb{N}$ such that $q_{b}\left(y_{2 n+1}, T u\right)>0$ for all $n \geq n_{2}$. By Lemma $6,0<q_{b}\left(y_{2 n+1}, T u\right) \leq H_{q_{b}}\left(S y_{2 n}, T u\right)$, so

$$
\begin{aligned}
& \max \left\{H_{q_{b}}\left(S y_{2 n}, T u\right), H_{q_{b}}\left(T u, S y_{2 n}\right), q_{b}\left(y_{2 n}, u\right),\right. \\
& \left.q_{b}\left(u, y_{2 n}\right)\right\}>0,
\end{aligned}
$$

for all $n \geq n_{2}$. By Lemma 6 , and $s \geq 1$, we get

$$
\begin{aligned}
\tau+ & F\left(s q_{b}\left(y_{2 n+1}, T u\right)\right) \leq \tau \\
& +\max \left\{F\left(s H_{q_{b}}\left(S y_{2 n}, T u\right)\right), F\left(s H_{q_{b}}\left(T u, S y_{2 n}\right)\right)\right\} .
\end{aligned}
$$

Now, $\rho_{s}^{*}\left(y_{2 n}, S y_{2 n}\right) \geq s$ and $\rho_{\mathrm{s}}^{*}(S u, u) \geq s$, and then by (64), we get

$$
\tau+F\left(s q_{b}\left(y_{2 n+1}, T u\right)\right) \leq F\left(Q_{b}\left(y_{2 n}, u\right)\right) .
$$

Since $F$ is strictly increasing, we have

$$
s q_{b}\left(y_{2 n+1}, T u\right)<Q_{b}\left(y_{2 n}, u\right) .
$$

Taking limit $n \longrightarrow \infty$, on both sides of inequality (106), we get

$$
\lim _{n \longrightarrow \infty} s q_{b}\left(y_{2 n+1}, T u\right)<\lim _{n \longrightarrow \infty} Q_{b}\left(y_{2 n}, u\right) .
$$

Since $q_{b}\left(u, S y_{2 n}\right) \leq q_{b}\left(u, y_{2 n+1}\right)$, taking limit $n \longrightarrow \infty$, on both sides, we get

$$
\lim _{n \longrightarrow \infty} q_{b}\left(u, S y_{2 n}\right)=0
$$

By using (64), we get

$$
\begin{aligned}
& Q_{b}\left(y_{2 n}, u\right) \\
& =\frac{q_{b}\left(y_{2 n}, y_{2 n+1}\right) q_{b}\left(y_{2 n}, T u\right)+q_{b}(u, T u) q_{b}\left(u, S y_{2 n}\right)}{\max \left\{q_{b}\left(y_{2 n}, T u\right), q_{b}\left(u, S y_{2 n}\right)\right\}} .
\end{aligned}
$$

Taking limit as $n \longrightarrow \infty$ and using inequality (108), we have

$$
\lim _{n \rightarrow \infty} Q_{b}\left(y_{2 n}, u\right)=\lim _{n \longrightarrow \infty} q_{b}\left(y_{2 n}, y_{2 n+1}\right)=0 \text {. }
$$

Now, inequality (107) implies

$$
\lim _{n \rightarrow \infty} s q_{b}\left(y_{2 n+1}, T u\right)<0
$$


Now

$$
q_{b}(u, T u) \leq s q_{b}\left(u, y_{2 n+1}\right)+s q_{b}\left(y_{2 n+1}, T u\right) .
$$

Taking limit as $n \longrightarrow \infty$,

$$
\begin{aligned}
q_{b}(u, T u) \leq & s \lim _{n \longrightarrow \infty} q_{b}(u, 2 n+1 \\
& +\lim _{n \longrightarrow \infty} s q_{b}\left(y_{2 n+1}, T u\right) .
\end{aligned}
$$

Using inequalities (85) and (111) in (113), we get

$$
q_{b}(u, T u)<0 .
$$

This is a contradiction, so $q_{b}(u, T u)=0$. Now assume that $q_{b}(T u, u)>0$, and then there exists $n_{3} \in \mathbb{N}$ such that $q_{b}\left(T u, y_{2 n+1}\right)>0$ for all $n \geq n_{3}$. By Lemma $6 q_{b}\left(T u, y_{2 n+1}\right) \leq$ $H_{q_{b}}\left(T u, S y_{2 n}\right)$, so

$$
\begin{aligned}
& \max \left\{H_{q_{b}}\left(S y_{2 n}, T u\right), H_{q_{b}}\left(T u, S y_{2 n}\right), q\left(y_{2 n}, u\right),\right. \\
& q_{b}\left(u, y_{2 n}\right)>0 .
\end{aligned}
$$

for all $n \geq n_{3}$. Following similar arguments as above, we get

$$
q_{b}(T u, u)<0 .
$$

So $q_{b}(T u, u)=0$. Hence $u \in T u$. As $\rho_{s}^{*}(S u, u) \geq s$ and $q_{b}(u, T u)=q_{b}(T u, u)=q_{b}(0,0)$, then Definition 14 implies

$$
\rho_{s}^{*}(u, S u) \geq s .
$$

Hence, the pair $(S, T)$ has a common fixed point $u$ in $\left(Y, q_{b}\right)$. Hence the proof is completed.

Corollary 18 (see [30]). Let $(X, d)$ be a complete metric space and $T: X \longrightarrow X$ be an F-Khan contraction. Then, $T$ has a unique fixed point $x^{*} \in X$ and for every $x \in X$ the sequence $\left\{T^{n} x\right\}$ converges to $x^{*}$.

\section{Single Valued Result with Application to System of Integral Equations}

Let $S, T: Y \longrightarrow Y$ be two self-mappings and $x_{0} \in Y$. Let $x_{1}=S x_{0}, x_{2}=T x_{1}, x_{3}=S x_{2}$ and so on. In this way, we construct a sequence $x_{n}$ in $X$ such that

$$
\begin{gathered}
x_{2 p+1}=S x_{2 p} \\
\text { and } x_{2 p+2}=T x_{2 p+1},
\end{gathered}
$$

(where $p=0,1,2, \ldots)$.

We say that $\left\{T S\left(x_{n}\right)\right\}$ is a sequence in $Y$ generated by $x_{0}$.

The following result is obtained by replacing the multivalued mappings with the single valued mappings in Theorem 10. Our result generalizes Theorem 24 in [41]. Also, we prove uniqueness of common fixed point in our result.

Theorem 19. Let $\left(Y, d_{q_{b}}\right)$ be a complete dislocated b-quasimetric space with constant $s \geq 1$ and $S, T: Y \longrightarrow$
$Y$ be two self-mappings. If there exists $F \in \mathscr{F}_{S}$ and $\tau, a>0$ such that for every two consecutive points $x, y$ belonging to the range of an iterative sequence $\left\{T S\left(y_{n}\right)\right\}$ with $\max \left\{d_{q b}(S x, T y), d_{q b}(T y, S x), D_{q b}(x, y), D_{q b}(y, x)\right\}>0$, we have

$$
\begin{aligned}
\tau+ & \max \left\{F\left(s d_{q b}(S x, T y)\right), F\left(s d_{q b}(T y, S x)\right)\right\} \\
& \leq \min \left\{F\left(D_{q_{b}}(x, y)\right), F\left(D_{q_{b}}(y, x)\right)\right\},
\end{aligned}
$$

where

$$
\begin{aligned}
& D_{q_{b}}(x, y)=\max \left\{d_{q b}(x, y),\right. \\
& \frac{d_{q b}(x, S x) \cdot d_{q b}(y, T y)}{a+\max \left\{d_{q b}(x, y), d_{q b}(y, x)\right\}}, d_{q b}(x, S x), \\
& \left.d_{q b}(y, T y)\right\},
\end{aligned}
$$

then $\left\{T S\left(y_{n}\right) \longrightarrow u \in X\right.$. Also, if $u$ satisfies (119), then $S$ and $T$ have a unique common fixed point $u$ in $X$ and $d_{q_{b}}(u, u)=0$.

Proof. Now, we have to prove uniqueness only. Let $x^{*}$ be another common fixed point of $S, T$. Suppose $d_{q b}\left(S u, T x^{*}\right)>$ 0 . Then, we have

$$
\begin{aligned}
& \tau+F\left(s d_{q b}\left(S u, T x^{*}\right)\right) \leq F\left(\operatorname { m a x } \left\{d_{q b}\left(u, x^{*}\right),\right.\right. \\
& \frac{d_{q b}(u, S u) \cdot d_{q b}\left(x^{*}, T x^{*}\right)}{1+\max \left\{d_{q b}\left(u, x^{*}\right), d_{q b}\left(x^{*}, u\right)\right\}}, d_{q b}(u, S u), \\
& \left.\left.d_{q b}\left(x^{*}, T x^{*}\right)\right\}\right)
\end{aligned}
$$

which implies that

$$
s d_{q b}\left(u, x^{*}\right)<d_{q b}\left(u, x^{*}\right)
$$

which is contradiction. Then $d_{q b}\left(S u, T x^{*}\right)=0$. Also

$$
\begin{aligned}
& \tau+F\left(s d_{q b}\left(S x^{*}, T u\right)\right) \leq F\left(\operatorname { m a x } \left\{d_{q b}\left(x^{*}, u\right),\right.\right. \\
& \\
& \quad \frac{d_{q b}\left(x^{*}, S x^{*}\right) \cdot d_{q b}(u, T u)}{1+\max \left\{d_{q b}\left(x^{*}, u\right), d_{q b}\left(u, x^{*}\right)\right\}}, d_{q b}\left(x^{*}, S x^{*}\right), \\
& \left.\left.d_{q b}(u, T u)\right\}\right),
\end{aligned}
$$

And then, we get $d_{q b}\left(S x^{*}, T u\right)=0$. So, $x^{*}=u$. Now, we deduce the following main result.

Corollary 20. Let $\left(Y, d_{q_{b}}\right)$ be a complete dislocated $b$ metric space with constant $s \geq 1$ and $S, T: Y \longrightarrow Y$ be 
two self-mappings. If there exists $F \in \mathscr{F}_{S}$ and $\tau, a>$ 0 such that for every two consecutive points $x, y$ belonging to the range of an iterative sequence $\left\{\operatorname{TS}\left(y_{n}\right)\right\}$ with $\max \left\{d_{q b}(S x, T y), D_{q b}(x, y)\right\}>0$, we have

$$
\tau+F\left(s d_{q b}(S x, T y)\right) \leq F\left(D_{q_{b}}(x, y)\right),
$$

where

$$
\begin{aligned}
& D_{q_{b}}(x, y)=\max \left\{d_{q b}(x, y),\right. \\
& \left.\quad \frac{d_{q b}(x, S x) \cdot d_{q b}(y, T y)}{a+d_{q b}(x, y)}, d_{q b}(x, S x), d_{q b}(y, T y)\right\}
\end{aligned}
$$

then $\left\{T S\left(y_{n}\right) \longrightarrow u \in X\right.$. Also, if $u$ satisfies (124), then $S$ and $T$ have a unique common fixed point $u$ in $X$ and $d_{q_{b}}(u, u)=0$.

Let $\mathscr{F}$ be the set of all functions $F: \mathbb{R}_{+} \longrightarrow \mathbb{R}$ defined by [21]. Then, we have the following new result.

Corollary 21. Let $\left(Y, d_{q_{b}}\right)$ be a complete dislocated quasimetric space and $S, T: Y \longrightarrow Y$ be two selfmappings. If there exists $F \in \mathscr{F}$ and $\tau, a>0$ such that for every two consecutive points $x, y$ belonging to the range of an iterative sequence $\left\{T S\left(y_{n}\right)\right\}$ with $\max \left\{d_{q b}(S x, T y), d_{q b}(T y, S x), D_{q b}(x, y), D_{q b}(y, x)\right\}>0$, we have

$$
\begin{aligned}
\tau+ & \max \left\{F\left(d_{q b}(S x, T y)\right), F\left(d_{q b}(T y, S x)\right)\right\} \\
& \leq \min \left\{F\left(D_{q_{b}}(x, y)\right), F\left(D_{q_{b}}(y, x)\right)\right\},
\end{aligned}
$$

where

$$
\begin{aligned}
& D_{q_{b}}(x, y)=\max \left\{d_{q b}(x, y),\right. \\
& \frac{d_{q b}(x, S x) \cdot d_{q b}(y, T y)}{a+\max \left\{d_{q b}(x, y), d_{q b}(y, x)\right\}}, d_{q b}(x, S x), \\
& \left.d_{q b}(y, T y)\right\}
\end{aligned}
$$

then $\left\{T S\left(y_{n}\right) \longrightarrow u \in X\right.$. Also, if $u$ satisfies (126), then $S$ and $T$ have a unique common fixed point $u$ in $X$ and $d_{q_{b}}(u, u)=0$.

Now, as an application, we discuss the application of Theorem 19 to find solution of the system of Volterra type integral equations. Consider the following integral equations:

$$
\begin{aligned}
& u(t)=\int_{0}^{t} K_{1}(t, s, u(s)) d s, \\
& v(t)=\int_{0}^{t} K_{2}(t, s, v(s)) d s
\end{aligned}
$$

for all $t \in[0,1]$. We find the solution of (128) and (129). Let $X=C\left([0,1], \mathbb{R}_{+}\right)$be the set of all continuous functions on
$[0,1]$, endowed with the complete dislocated $b$-quasi-metric. For $u \in C\left([0,1], \mathbb{R}_{+}\right)$, define supremum norm as $\|u\|_{\tau}=$ $\sup _{t \in[0,1]}\left\{u(t) e^{-\tau t}\right\}$, where $\tau>0$ is taken arbitrarily. Then define

$$
\begin{aligned}
d_{\tau}(u, v) & =\left[\sup _{t \in[0,1]}\left\{(u(t)+2 v(t)) e^{-\tau t}\right\}\right]^{2} \\
& =\|u+2 v\|_{\tau}^{2}
\end{aligned}
$$

for all $u, v \in C\left([0,1], \mathbb{R}_{+}\right)$, and with these settings, $\left(C\left([0,1], \mathbb{R}_{+}\right), d_{\tau}\right)$ becomes a dislocated $b$-quasi-metric space.

Now we prove the following theorem to ensure the existence of solution of integral equations.

Theorem 22. Assume the following conditions are satisfied:

(i) $K_{1}, K_{2}:[0,1] \times[0,1] \times \mathbb{R}_{+} \longrightarrow \mathbb{R}_{+}$and $f, g:[0,1] \longrightarrow$ $\mathbb{R}_{+}$are continuous.

(ii) Define

$$
\begin{aligned}
& S u(t)=\int_{0}^{t} K_{1}(t, s, u(s)) d s \\
& T v(t)=\int_{0}^{t} K_{2}(t, s, v(s)) d s .
\end{aligned}
$$

Suppose there exist $\tau>1$, such that

$$
\begin{aligned}
\max & \left\{K_{1}(t, s, u)+2 K_{2}(t, s, v), K_{2}(t, s, v)\right. \\
& \left.+2 K_{1}(t, s, u)\right\} \\
& \leq \sqrt{\tau e^{2 \tau s-\tau} \min \{M(u, v), M(v, u)\}}
\end{aligned}
$$

for all $t, s \in[0,1]$ and $u, v \in C([0,1], \mathbb{R})$, where

$M(u, v)$

$$
=\max \left\{\begin{array}{c}
\|u+2 v\|^{2}, \frac{\|u+2 S u\|^{2}\|v+2 T v\|^{2}}{a+\max \left\{\|u+2 v\|^{2},\|v+2 u\|^{2}\right\}}, \\
\|u+2 S u\|^{2},\|v+2 T v\|^{2}
\end{array}\right\} .
$$

Then integral equations (128) and (129) have a unique solution.

Proof. By assumption (ii) and (132), we have

$$
\begin{aligned}
& \max \{S u+2 T v, T v+2 S u\} \\
& =\max \left\{\int_{0}^{t}\left(K_{1}(t, s, u)+2 K_{2}(t, s, v)\right) d s\right. \\
& \left.\int_{0}^{t}\left(K_{2}(t, s, v)+2 K_{1}(t, s, u)\right) d s\right\} \\
& \leq \int_{0}^{t} \sqrt{\tau e^{2 \tau s-\tau} \min \{M(u, v), M(v, u)\}} d s \\
& (\max \{S u+2 T v, T v+2 S u\})^{2} \leq \tau e^{-\tau} \min \{M(u, v), \\
& M(v, u)\} \int_{0}^{t} e^{2 \tau s} d s \leq \frac{1}{2} e^{-\tau} \min \{M(u, v), M(v, u)\} \\
& \cdot e^{2 \tau t} .
\end{aligned}
$$


This implies

$$
\begin{aligned}
& \left(\max \{S u+2 T v, T v+2 S u\} e^{-\tau t}\right)^{2} \\
& \leq \frac{1}{2} e^{-\tau} \min \{M(u, v), M(v, u)\} .
\end{aligned}
$$

That is,

$$
\begin{aligned}
& 2\|\max \{S u+2 T v, T v+2 S u\}\|_{\tau}^{2} \\
& \quad \leq e^{-\tau} \min \{M(u, v), M(v, u)\},
\end{aligned}
$$

which further implies

$$
\begin{aligned}
\tau+ & 2 \ln \|\max \{S u+2 T v, T v+2 S u\}\|_{\tau}^{2} \\
& \leq \ln \min \{M(u, v), M(v, u)\}, \\
\tau+ & \left.\max \left\{s \ln \|S u+2 T v\|_{\tau}^{2}, s \ln \| T v+2 S u\right\} \|_{\tau}^{2}\right\} \\
& \leq \ln \min \{M(u, v), M(v, u)\} .
\end{aligned}
$$

So, all the conditions of Theorem 19 are satisfied for $(a)=\ln a$, $d_{\tau}(u, v)=\|u+2 v\|_{\tau}^{2}, s=2$. Hence integral equations given in (128)and (129) have a common unique solution.

Remark 23. By setting different values of $M(u, v)$ in (132), we can obtain different weak contractive inequalities and results as corollaries of Theorem 22.

\section{Conclusion}

In this work, we have discussed the notion of dislocated $b$-quasi-metric space and given an application to find the solutions of the nonlinear integral equations in such spaces. New results in b-quasi-metric, quasi-metric, quasi dislocated metric, dislocated metric, and metric can be obtained as corollaries of our theorems, which are still not present in the literature. The notions of $\rho_{s}^{*}$-Alt multivalued mapping and $F \rho_{s}^{*}$ Khan type contraction on a sequence have been introduced. Our observation is that the fixed points of mappings which are contractive only on a sequence can be ensured by the fixed point results. Our results extend the results given in $[41,45]$.

\section{Data Availability}

No data were used to support this study.

\section{Conflicts of Interest}

The authors declare that they have no competing interests.

\section{Acknowledgments}

This article was partially supported by the Department of Mathematics and Statistics, Riphah International University, Islamabad, Pakistan. This is a part of research work done by $\mathrm{PhD}$ student, Shaif Saleh Alshoraify, under the supervision of second author. Article processing charges will be shared by the brother of Shaif Saleh Alshoraify from Yemen.

\section{References}

[1] A. B. Amar, A. Jeribi, and M. Mnif, "Some fixed point theorems and application to biological model," Numerical Functional Analysis and Optimization, vol. 29, no. 1-2, pp. 1-23, 2008.

[2] A. Jeribi, B. Krichen, and B. Mefteh, "Existence of solutions of a two-dimensional boundary value problem for a system of nonlinear equations arising in growing cell populations," Journal of Biological Dynamics, vol. 7, no. 1, pp. 218-232, 2013.

[3] K. Leibovic, "The principle of contration mapping in nonlinear and adoptive controle systems," IEEE Transactions on Automatic Control, vol. 9, pp. 393-398, 1964.

[4] G. Medrano-cerda, "A fixed point formulation to parameter estimation problems," in Proceedings of the 26th IEEE Conference on Decision and Control, pp. 1914-1915, The University of California, Los Angeles, Calif, USA, December 1987.

[5] S. Czerwik, "Nonlinear set-valued contraction mappings in b-metric spaces," Atti del Seminario Matematico e Fisico dell'Università di Modena, vol. 46, no. 2, pp. 263-276, 1998.

[6] M. U. Ali, T. Kamran, and M. Postolache, "Solution of Volterra integral inclusion in b-metric spaces via new fixed point theorem," Nonlinear Analysis: Modelling and Control, vol. 2017, no. 1, pp. 17-30, 2017.

[7] Q. Mahmood, A. Shoaib, T. Rasham, and M. Arshad, "Fixed point results for the family of multivalued f-contractive mappings on closed ball in complete dislocated b-metric spaces," Mathematics, vol. 7, no. 1, p. 56, 2019.

[8] T. Rasham, A. Shoaib, N. Hussain, B. A. S. Alamri, and M. Arshad, "Multivalued fixed point results in dislocated b- metric spaces with application to the system of nonlinear integral equations," Symmetry, vol. 11, no. 1, p. 40, 2019.

[9] X. Zhao, F. He, and Y. Sun, "Common fixed point results for quasi-contractions of Ciric type in b-metric spaces with Qtfunctions," Journal of Nonlinear Functional Analysis, vol. 2017, article 43, pp. 1-18, 2017.

[10] M. Arshad, Z. Kadelburg, S. Radenovic, A. Shoaib, and S. Shukla, "Fixed points of $\alpha$-dominated mappings on dislocated quasi metric spaces," Filomat, vol. 31, no. 11, pp. 3041-3056, 2017.

[11] I. Beg, M. Arshad, and A. Shoaib, "Fixed point on a closed ball in ordered dislocated quasi metric spaces," Fixed Point Theory, vol. 16, no. 2, pp. 195-206, 2015.

[12] A. Shoaib, "Fixed point results for $\alpha^{\star}-\psi$-multivalued mappings," Bulletin of Mathematical Analysis and Applications, vol. 8, no. 4, pp. 43-55, 2016.

[13] A. Shoaib, M. Arshad, T. Rasham, and M. Abbas, "Unique fixed points results on closed ball for dislocated quasi G-metric spaces," Transactions of A. Razmadze Mathematical Institute, vol. 171, no. 2, pp. 221-230, 2017.

[14] M. A. Alghamdi, N. Hussain, and P. Salimi, "Fixed point and coupled fixed point theorems on $b$-metric-like spaces," Journal of Inequalities and Applications, vol. 2013, article 402, 2013.

[15] I. A. Bakhtin, "The contraction mapping principle in almost metric space," Functional Analysis and its Applications, vol. 30, pp. 26-37, 1989.

[16] A. Felhi, S. Sahmim, and H. Aydi, "Ulam-hyers stability and well-posedness of fixed point problems for $\alpha$ - $\lambda$-metric spaces," Fixed Point Theory and Applications, vol. 2016, no. 1, 20 pages, 2016.

[17] H.-C. Wu, "Coincidence point and common fixed point theorems in the product spaces of quasi-ordered metric spaces," Journal of Nonlinear and Variational Analysis, vol. 1, no. 2, pp. 175-199, 2017. 
[18] C. Klin-eam and C. Suanoom, "Dislocated quasi-b-metric spaces and fixed point theorems for cyclic contractions," Fixed Point Theory and Applications, vol. 2015, article 74, 2015.

[19] M. Abbas, V. Rakocevic, and B. Leyew, "Common fixed points of $(\alpha-\psi)$ - generalized rational multivalued contractions in dislocated quasi b-metric spaces and applications," Filomat, vol. 31, no. 11, pp. 3263-3284, 2017.

[20] M. U. Rahman and M. Sarwar, "Dislocated quasi b-metric space and fixed point theorems," Electronic Journal of Mathematical Analysis and Applications, vol. 4, no. 2, pp. 16-24, 2016.

[21] D. Wardowski, "Fixed points of a new type of contractive mappings in complete metric spaces," Fixed Point Theory and Applications, vol. 2012, article 94, 2012.

[22] Ö. Acar and I. Altun, "A fixed point theorem for multivalued mappings with $\delta$-distance," Abstract and Applied Analysis, vol. 2014, Article ID 497092, 5 pages, 2014.

[23] Ö. Acar, G. Durmaz, and G. Minak, "Generalized multivalued F-contractions on complete metric spaces," Bull. Iranian Math. Society, vol. 40, no. 6, pp. 1469-1478, 2014.

[24] J. Ahmad, A. Al-Rawashdeh, and A. Azam, "New fixed point theorems for generalized F-contractions in complete metric spaces," Fixed Point Theory and Applications, vol. 2015, no. 1, article no. 80, 2015.

[25] M. Arshad, S. U. Khan, and J. Ahmad, "Fixed point results for Fcontractions involving some new rational expressions," Journal of Fixed Point Theory and Applications, vol. 11, no. 1, pp. 79-97, 2016.

[26] N. Hussain, J. Ahmad, and A. Azam, "On Suzuki-Wardowski type fixed point theorems," Journal of Nonlinear Sciences and Applications, vol. 8, no. 6, pp. 1095-1111, 2015.

[27] A. Hussain, M. Arshad, and S. U. Khan, " $\tau$-generalization of fixed point results for F-contraction," Bangmod International Journal of Mathematical \& Computational Science, vol. 1, no. 1, pp. 127-137, 2015.

[28] S. U. Khan, M. Arshad, A. Hussain, and M. Nazam, "Two new types of fixed point theorems for F-contraction," Journal of Advanced Studies in Topology, vol. 7, no. 4, pp. 251-260, 2016.

[29] E. Karap nar, H. Piri, and H. H. Alsulami, "Fixed points of modified F-contractive mappings in complete metric-like spaces," Journal of Function Spaces, vol. 2015, Article ID 270971, 9 pages, 2015.

[30] H. Piri, S. Rahrovi, H. Marasi, and P. Kumam, "Fixed point theorem for F-Khan-contractions on complete metric spaces and application to the integral equations," Journal of Nonlinear Sciences and Applications, vol. 10, no. 9, pp. 4564-4573, 2017.

[31] J. Nadler, "Multi-valued contraction mappings," Pacific Journal of Mathematics, vol. 30, pp. 475-488, 1969.

[32] H. Aydi, M.-F. Bota, E. Karapinar, and S. Mitrović, "A fixed point theorem for set-valued quasi-contractions in b-metric spaces," Fixed Point Theory and Applications, vol. 2012, article 88, 2012.

[33] I. Beg, A. R. Butt, and S. Radenović, "The contraction principle for set valued mappings on a metric space with a graph," Computers \& Mathematics with Applications, vol. 60, no. 5, pp. 1214-1219, 2010.

[34] A. Shoaib, A. Hussain, M. Arshad, and A. Azam, "Fixed point results for $\alpha^{\star}-\psi$-Ciric type multivalued mappings on an intersection of a closed ball and a sequence with graph," Journal of Mathematical Analysis, vol. 7, no. 3, pp. 41-50, 2016.

[35] A. Shoaib, A. Azam, M. Arshad, and A. Shahzad, "Fixed point results for the multivalued mapping on closed ball in dislocated fuzzy metric space," Journal of Mathematical Analysis, vol. 8, no. 2, pp. 98-106, 2017.
[36] A. Shoaib, A. Azam, and A. Shahzad, "Common fixed point results for the family of multivalued mappings satisfying contractions on a sequence in Hausdorff fuzzy metric space," Journal of Computational Analysis and Applications, vol. 24, no. 4, pp. 692-699, 2018.

[37] J. Aubin, Mathematical Methods of Game and Economic Theory, North-Holland Publishing, Amsterdam, The Netherlands, 1979.

[38] S. Bohnenblust and S. Karlin, Contributions to the Theory of Games, Princeton University Press, Princeton, NJ, USA, 1950.

[39] S. Carl and S. Heikkila, Fixed Point Theory in Ordered Sets and Applications: From Differential and Integral Equations to Game Theory, Springer, New York, NY, USA, 2010.

[40] J. Li, "Several extensions of the Abian-Brown fixed point theorem and their applications to extended and generalized Nash equilibria on chain-complete posets," Journal of Mathematical Analysis and Applications, vol. 409, no. 2, pp. 1084-1092, 2014.

[41] T. Rasham, A. Shoaib, N. Hussain, M. Arshad, and S. U. Khan, "Common fixed point results for new Ciric-type rational multivalued F-contraction with an application," Journal of Fixed Point Theory and Applications, vol. 20, no. 1, article 45, 16 pages, 2018.

[42] H. Piri, S. Rahrovi, and P. Kumam, "Generalization of Khan fixed point theorem," Journal of Mathematics and Computer Science, vol. 17, no. 1, pp. 76-83, 2017.

[43] M. S. Khan, "A fixed point theorem for metric spaces," Rendiconti dell'Istituto di Matematica dell'Università di Trieste, vol. 8, no. 1, pp. 69-72, 1976.

[44] B. Fisher, "On a theorem of Khan," Rivista di Matematica della Università di Parma, vol. 4, pp. 135-137, 2016.

[45] I. Altun, N. Al Arifi, M. Jleli, A. Lashin, and B. Samet, "A new approach for the approximations of solutions to a common fixed point problem in metric fixed point theory," Journal of Function Spaces, vol. 2016, Article ID 6759320, 5 pages, 2016. 


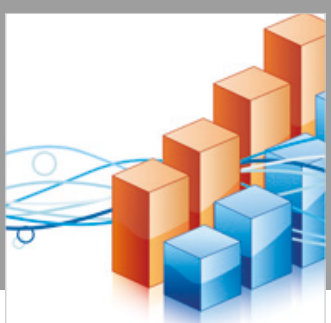

Advances in

Operations Research

\section{-n-m}
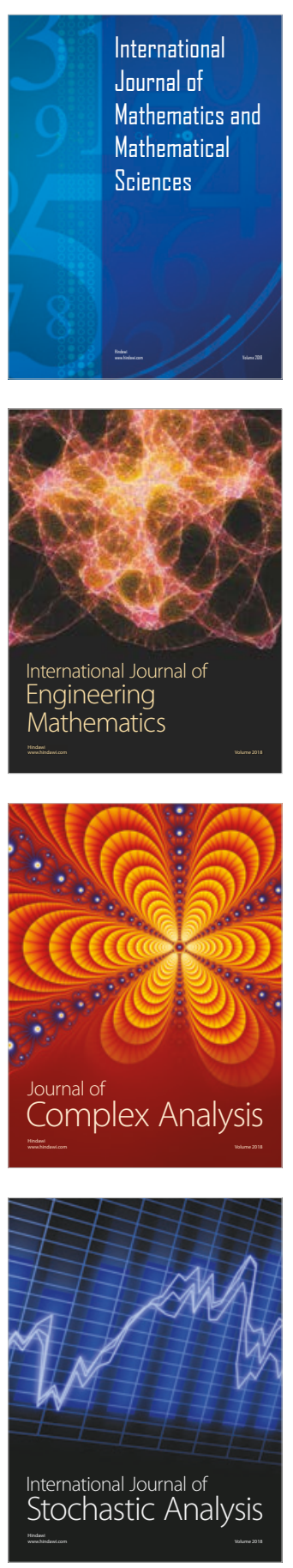
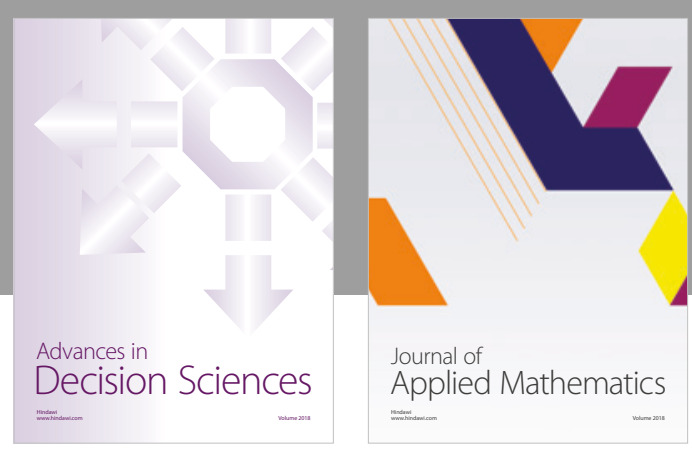

Journal of

Applied Mathematics
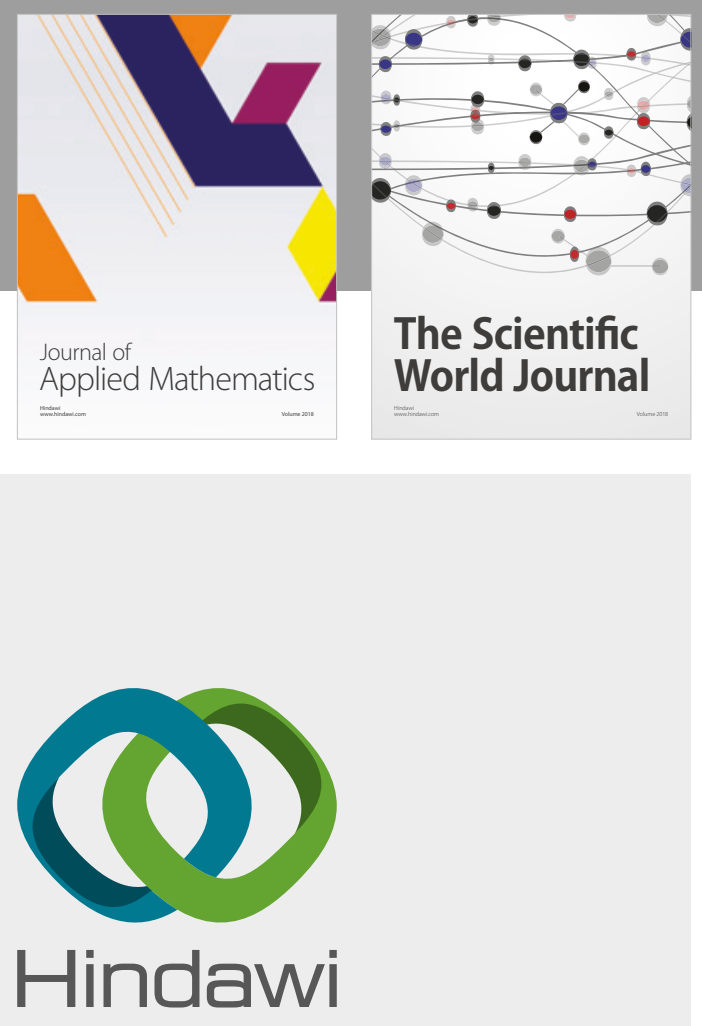

Submit your manuscripts at

www.hindawi.com

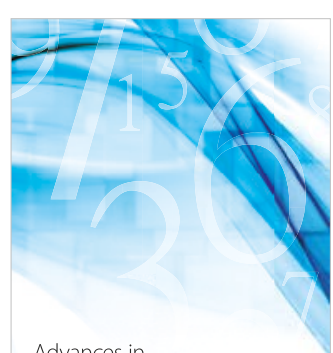

Advances in
Numerical Analysis
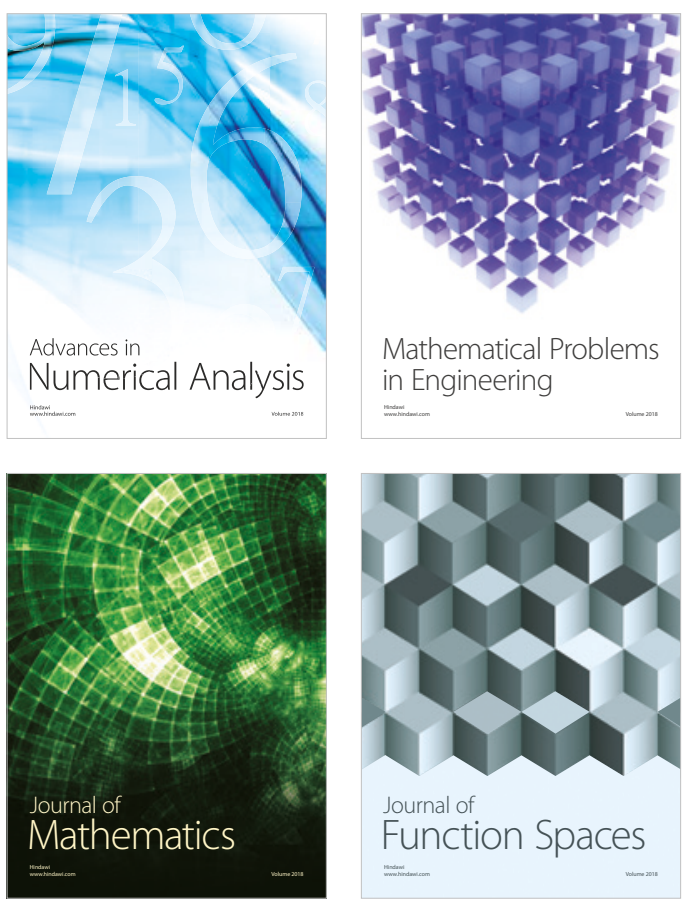

Mathematical Problems in Engineering

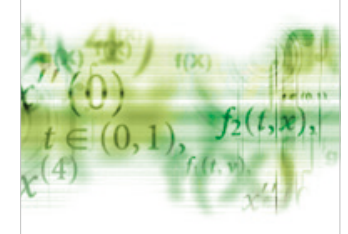

International Journal of

Differential Equations

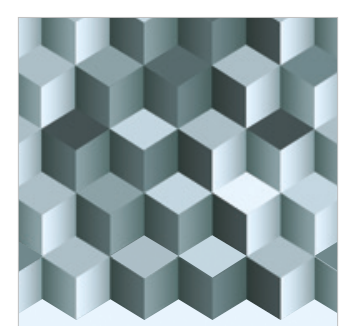

Journal of

Function Spaces

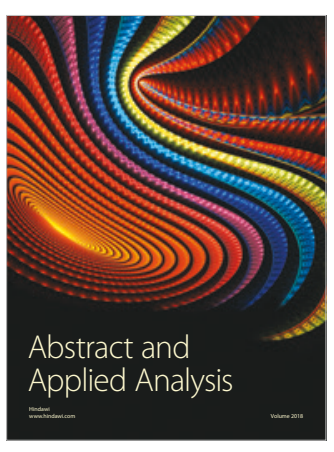

The Scientific

World Journal

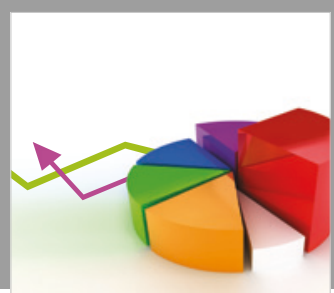

Journal of

Probability and Statistics
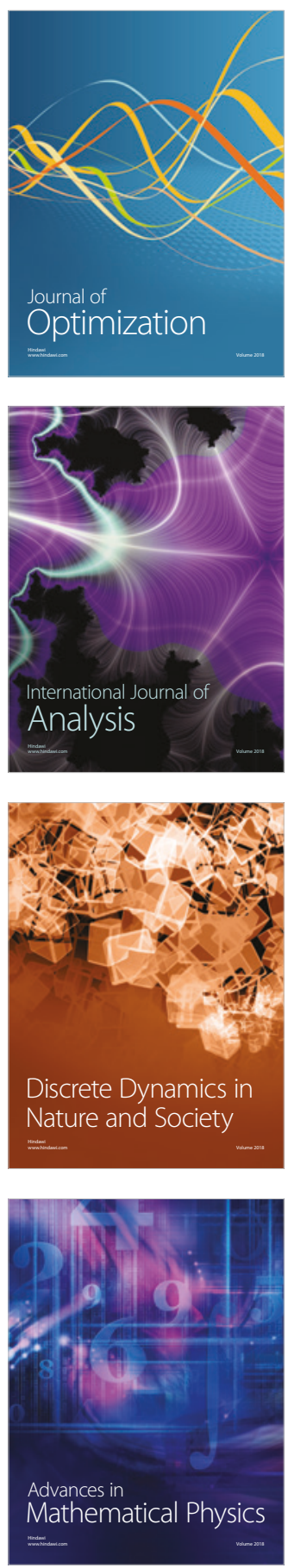